\title{
1. Advanced turbulence models and boundary $=$ conditions for flows around different configurations of ground-mounted buildings
}

\author{
Riccardo Longo*,a , Marco Ferrarottia ${ }^{\text {, Clara García Sánchez }}{ }^{\mathrm{b}, \mathrm{c}, \mathrm{d}}$, Marco \\ Derudie ${ }^{\text {, Alessandro Parente }}{ }^{*}$ a \\ aUniversité Libre de Bruxelles - Aéro-Thermo-Mécanique Department \\ bUniversity of Antwerp - EMAT, Department of Physics \\ ${ }^{c}$ Von Karman Institute for Fluid Dynamics \\ ${ }^{d}$ Stanford University - Department of Civil and Environmental Engineering \\ e Politecnico di Milano - Dipartimento di Chimica, Materiali ed Ingegneria Chimica "G. Natta"
}

\section{${ }_{11}$ Abstract}

12 When dealing with Atmospheric Boundary Layer ( $A B L)$ simulations, commer-

13 cial computational fluid dynamics (CFD) acquires a strategic resonance. Thanks to its good compromise between accuracy of results and calculation time, RANS still represents a valid alternative to more resource-demanding methods. However, focusing on the models' performances in urban studies, LES generally outmatches RANS results, even if the former is at least one order of magnitude more expensive. Consequently, the present work aims to propose a variety of approaches meant to solve some of the major problems linked to RANS simulations and to further improve its accuracy in typical urban contexts. All of these models are capable of switching from an undisturbed flux formulation to a disturbed one through a local deviation or a marker function. For undisturbed flows, a comprehensive approach is adopted, solving the issue of the erroneous stream-wise gradients affecting the turbulent profiles. Around obstacles, Non-Linear Eddy-Viscosity closures are adopted, due to their prominent capa-

*Corresponding author’s Email: riccardo.longo@mail.polimi.it; Alessandro.Parente@ulb.ac.be 
bility in capturing the anisotropy of turbulence. The purpose of this work is then to propose a new Building Influence Area concept and to offer more affordable alternatives to LES simulations without sacrificing a good grade of accuracy.

\section{Keywords}

Atmospheric Boundary Layer; Building Influence Area; Comprehensive Approach; Improved $k-\epsilon$ model; Marker function; Non-linear Eddy-viscosity models.

\section{Introduction}

Atmospheric boundary layer simulation over complex terrains (both in rural and urban contexts) is a crucial juncture for the correct estimation of flow-field in urban canopy; wind load on turbines and buildings; and pollutant dispersion. It is also employed for the safe siting of facilities manufacturing or dealing with hazardous gases. Within this context, the forecast accuracy is of paramount importance to draw conclusions that can support policy maker decisions. In recent years, these specific subjects have been examined and studied mostly through Reynolds-Averaged Navier-Stokes by several research groups (i.e. Castro et al. [8] 2003; Blocken et al. [5] 2007; Pontiggia et al. [29] 2009; Balogh et al. [1] 2012; Parente et al. [24] 2017. As demonstrated by Xie and Castro [39] (2006), Large Eddy Simulation (LES) can offer improved performance for $\mathrm{ABL}$ flows, provided an acceptable characterisation of the inflow conditions. However, due to the large scales encompassed by ABL flows, LES methods are considerably more honerous than RANS (Rodi [35] 1997). Consequently, simulations of $A B L$ flows are often carried out using RANS in conjuction with two-equation turbulence models, with the aim of providing fast and feasible 
answers to the various design requests. That notwithstanding, there are two non-negligible drawbacks linked to RANS simulations: the well-known horizontal inhomogeneity affecting the profiles, and the inconsistency between wall functions and turbulence models. Blocken et al. [5] (2007) and, subsequently, O'Sullivan et al. [37] (2011) further improved the original Richards and Hoxey [31] (1993) near-wall treatment. They also focused on how excessive streamwise gradients can be influenced by an inappropriate wall-function formulation, as well as roughness height and boundary conditions. When taking into account the decrease of shear stress together with height, the horizontal inhomogeneity was quantitatively estimated by Juretic and Kozmar [20]. Recently, Gorlé et al. [15] introduced a new formulation for the $C_{\mu}$ constant, and for the turbulent dissipation Prandtl number, $\sigma_{\epsilon}$, in order to achieve homogeneity with the $k$ profile proposed by Yang et al. [41]. An analogous approach is further validated and extended in Parente and Benocci [27] (2010), through a proper modification of the $k-\epsilon$ turbulence model according to the set of inlet conditions by Yang et al. [41]. This turbulent kinetic energy definition also proved to be valid for accurate modelling of the atmospheric dispersion, i.e. Riddle et al. (2004) [33], Pontiggia et al. [29] (2009), Gorlé et al. [15] (2009). The restriction of the former approach is represented by the unsatisfactory inlet profile adopted for turbulent kinetic energy which is not able to satisfy all the governing simulations involved in the problem. As a consequence, Parente et al. [25] [26] (2011) proposed a comprehensive approach consisting of a new set of fully developed inlet turbulent conditions for the neutral $A B L$. As an alternative, Yan et al. [40] (2016) developed a modelling methodology for the simulation of horizontally homogeneous flows, with the adoption of an arbitrary shear stress approach inside the RNG $k-\epsilon$ model. As for the correct representation of the turbulence properties in disturbed flows (namely in the vicinity of obstacles), a 
building influence area (BIA) has been developed [25] and further perfected in the last few years. Such a transition is generally referred to as "blending" and inside the BIA, specific turbulence models are applied.

Despite these remarkable improvements, the modelling accuracy of the flowfield around bluff bodies, where the standard two-equation turbulence models keep on failing (Durbin [10] 1996 ), still remains problematic and, at least, a challenging task. First of all, this kind of flow-field is quite sensitive to the incoming boundary layer properties, as stated by Porté-Agel et al. [30] (2014). Moreover, correct prediction of the size, shape and position of the separation bubble on the building and of the recirculation/stagnation zones - both upwind and in the wake - is not straightforward (Gorlé [17] 2010). In order to firmly improve the performance of the standard two eqs. models in proximity of obstacles, one possible path is to adopt higher order term closures for the stressstrain relation. Different quadratic stress-strain relations have been proposed to improve the applicability of linear eddy-viscosity models at an acceptable computational cost (Shih et al. [36] (1993) ). However, different comparisons proved that no one quadratic relation guarantees significant improvement in performance. Following this trend, Craft et al. [9] (1996) proposed a cubic relation between the strain-rate and vorticity tensors and the stress tensor, which behaves much better than an ordinary eddy-viscosity model, being also able to properly reproduce the effects of stream-line curvature. According to the same recursive cubic formulation, Lien et al. [21] (1996) and Ehrhard et al. [11] (2000), also edited and tuned this type of model through a proper definition of the coefficients for the non-linear terms.

Merci et al. [23] further investigated cubic models, proposing a new formulation for the non-linear closure. Furthermore he claimed $C_{\mu}$ to be the only relevant parameter - especially in respect to the non-linear coefficients - for all the flows 
characterized by reduced swirl and vorticity.

The present paper, moving from an assessed verification of the proposed turbulence models in open-field simulations, is centred around both the CEDVAL A1-1 (displaying a scaled single ground-mounted building, as shown in Figure 1)on the left) and the CEDVAL B1-1 (displaying an array of $7 \times 3$ A1-1 buildings, on the right) test cases available from the BLASIUS Wind Tunnel of the Environmental Wind Tunnel Laboratory of the Meteorological Institute of Hamburg University [43].
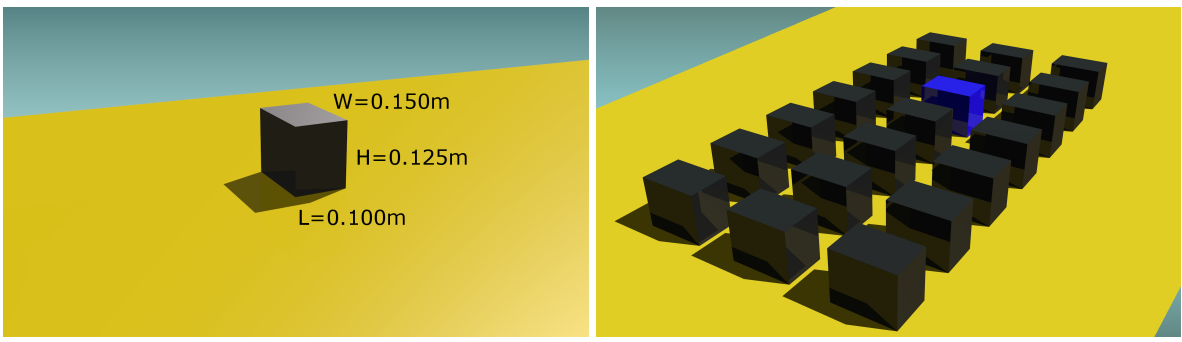

Figure 1: Cedval A1-1 (on the left) and B1-1 (on the right) test cases, rendered in Blender

As a consequence, it focuses on the topical challenges linked to the flowfield simulation in a typical urban context.

The aim of this study is somewhat multifaceted, but the main targets are:

- to demonstrate the relevance of using a Building Influence Area both for improved results and for reducing the computational resources required all over the domain;

- to further improve the detection of an obstacle and to investigate the effect of the BIA definition on the results;

- to develop a new Building Influence Area formulation based on a marker which measures the local deviation from a parallel shear flow;

- to employ different NLEV (non-linear eddy-viscosity) closures with the 
aim of investigating the influence of both the modified value of $C_{\mu}$ and the non-linear terms;

- to finally point out which model combination results in a better representation of the ground and obstacles' influences on the flow-field.

\section{Governing equations and implementation}

In RANS simulations fully developed profiles of velocity and turbulence characteristics are generally imposed. As previously mentioned, a crucial problem witnessed when applying RANS methodologies to ABL flows, deeply related to a proper selection of boundary conditions, is represented by the undesired changes (stream-wise gradients) that occur in the vertical profiles of mean wind speed and turbulence quantities as they travel from the inlet of the computational domain to the outlet.

This problem has been described in detail (Blocken et al., 2007 [5]) and it can dramatically affect the overall quality of the simulations.

\subsection{Comprehensive $k-\epsilon$ model}

Typically, inlet profiles of mean longitudinal velocity and turbulent properties under neutral stratification conditions are defined according Richards and Hoxey (1993) [31] formulation:

$$
U=\frac{u_{*}}{\kappa} \ln \left(\frac{z+z_{0}}{z_{0}}\right),
$$

$$
k=\frac{u_{*}^{2}}{\sqrt{C_{\mu}}},
$$

$$
\epsilon=\frac{u_{*}^{3}}{\kappa\left(z+z_{0}\right)} \text {. }
$$


In order to make eqs. (1)-(3) analytical solutions of the standard $k-\epsilon$ model, following Pontiggia et al. (2009) [29] and Parente et al. (2011) [25], the following source term has to be added to the dissipation rate eq.:

$$
S_{\epsilon}(z)=\frac{\rho u_{*}^{4}}{\left(z+z_{0}\right)^{2}}\left(\frac{\left(C_{\epsilon 2}-C_{\epsilon 1}\right) \sqrt{C_{\mu}}}{\kappa^{2}}-\frac{1}{\sigma_{\epsilon}}\right) .
$$

This source term can represent both an increment $\left(S_{\epsilon}>0\right)$ or a reduction $\left(S_{\epsilon}<0\right)$ of the turbulent dissipation rate due to peculiar atmospheric features that the standard $k-\epsilon$ model is typically not able to reproduce. Gorlé et al. (2009) [15] , considering the experimental decay of $k$ with height, proposed a generalization of the expression of $C_{\mu}$ as a function of $z$, obtaining:

$$
C_{\mu}=\frac{u_{*}^{4}}{k^{2}}
$$

The consinstency of the functional form of $C_{\mu}$, once using the inlet profiles proposed by Yang et al. [41] (2009) is guaranteed throughout the introduction of an additional source term for the $k$ transport eq. (Parente et al. [25] 2011). One additional approach aims at deriving a new profile which is solution of the turbulent kinetic energy transport eq., considering the functional variation of $C_{\mu}$. Once assumed local equilibrium between turbulence production and dissipation, employing the analytical expression of the inlet velocity profile and integrating the so-obtained eq., the following general solution for turbulent kinetic energy profile is then achieved:

$$
k(z)=C_{1} \ln \left(z+z_{0}\right)+C_{2} .
$$

$C_{1}$ and $C_{2}$ are constants determined by fitting the equations to the measured profiles of $k$. The full set of inlet conditions and turbulence model formulation can be summarized in Table 1. This set of inlet boundary conditions, once 


\begin{tabular}{ll}
\hline Inlet Conditions & Turbulence Model \\
\hline$U=\frac{u_{*}}{\kappa} \ln \left(\frac{z+z_{0}}{z_{0}}\right)$ & $\mu_{t}=C_{\mu} \rho \frac{k^{2}}{\epsilon}$ \\
$k(z)=C_{1} \ln \left(z+z_{0}\right)+C_{2}$ & $S_{\epsilon}(z)=\frac{\rho u_{*}^{4}}{\left(z+z_{0}\right)^{2}}\left(\frac{\left(C_{\epsilon 2}-C_{\epsilon 1}\right) \sqrt{C_{\mu}}}{\kappa^{2}}-\frac{1}{\sigma_{\epsilon}}\right)$ \\
$\epsilon(z)=\frac{u_{*}^{3}}{\kappa\left(z+z_{0}\right)}$ & $C_{\mu}=\frac{u_{*}^{4}}{k^{2}}$ \\
\hline
\end{tabular}

Table 1: Set of Inlet conditions and turbulence model formulation, also referred to as the "Comprehensive approach", able to guarantee the desired homogeneity of turbulence properties in the computational domain.

coupled with appropriate wall functions, represents a consistent extension of the formulation proposed by Richards and Hoxey (1993) [31] to the case of a non-constant turbulent kinetic energy profile. It is able to remove the horizontal inhomogeneity, improving the two eqs. model performance when dealing with open-field simulations.

The turbulence model formulation and the turbulence dissipation rate source term $S_{\epsilon}(z)$ are not any-more effective when dealing with obstacles or complex orographies. Despite the relative simple geometrical configuration of a rectangular building, the corresponding flow-field is quite complex with strong pressure gradients, streamline curvature and multiple, unsteady separation regions. These phenomena are obviously accentuated once dealing with the array of buildings test case. When the wind hits a rectangular building, a "separation bubble" develops on the top of the structure, starting from the leading edge. Moreover, a "horseshoe-type" vortex is engendered in the separation region in the front and it is bent around the cube. On the leeward side of the building, another large separation zone is created as the wind flows over its back edge. The building wake can extend further downstream (up to 20 times [42]) and is characterized by increased turbulence and reduced wind speed. The main guide-line of this study will be the initial implementation and testing 
of a proper turbulence approach for disturbed flows on the single building (A11) case. Subsequently, once assessed the model capabilities, it will be further validated on the more challenging array of buildings (B1-1) test-case.

\subsection{BIA based on local turbulent properties deviation}

In a first step, in order to better reproduce the disturbed flow-field, Gorlé et al. (2009) [15] proposed an alteration of the turbulence model parameters $C_{\mu}$ and $\sigma_{\epsilon}$ inside a "Building Influence Area" (BIA) which, according to Beranek (1979) [4], is defined as a half sphere ( $P S 1$ configuration) incorporating the obstacle. Parente et al. (2011) [25] further analysed the effect of the BIA size and shape constraining its area to the region above and downstream the building ( $P S 2$ configuration). The main issue of both the PS1 and $P S 2$ configurations is that they define an "a priori" region with an abrupt transition, where the $A B L$ does not hold, leading to the problem of neglecting the real nature of the flow-field. Consequently Parente et al. (2011) [25] suggested an approach for the automatic detection of the BIA, permitting a gradual transition of the turbulence model parameters from the formulation proposed for the undisturbed $A B L$ to one more suitable for immediately upwind and wake flow regions. In order to achieve this blending process, a local deviation from the undisturbed $\mathrm{ABL}$ conditions is then introduced. This one, considering a local relative turbulent property difference, automatically identifies the extent of the flow region affected by the obstacle. The first blending formulation proposed takes into account the relative velocity difference between a homogeneous $A B L$ and the disturbed flow regions (Parente et al. [24]), while the subsequent formulation is related to turbulent kinetic energy. Both approaches belong to the so-called Pure blending formulation and they consider the ABL properties (velocity $u$, or turbulent kinetic energy $k$ ) for the deviation only one by one. Following the 
aim of exploiting the strong points of both approaches, improving the detection of the disturbed flow-field, a new formulation is proposed in this study, under the name of Hybrid Blending, based on a fruitful combination of the two pure blending formulas. Namely, $u$ and $k$ are simultaneously taken into account to compute a deviation term, by locally selecting the maximum between the values obtained using $u$ and $k$ individually. This is briefly shown in Table 2 .

Table 2: Formulation of the blending metric for the pure and hybrid blending approaches

\begin{tabular}{ccc}
\hline \multicolumn{2}{c}{ Pure blending } & Hybrid blending \\
\hline $\mathrm{V}$ & TKE & V \& TKE \\
$\delta_{u}=\min \left[\left|\frac{u-u_{A B L}}{u_{A B L}}\right|, 1\right] \quad \delta_{k}=\min \left[\left|\frac{k-k_{A B L}}{k_{A B L}}\right|, 1\right]$ & $\delta_{h}=\max \left[\delta_{u}, \delta_{k}\right]$ \\
\hline
\end{tabular}

\subsection{BIA based on local deviation from a parallel shear flow}

A new formulation for detecting the disturbed area around an obstacle is derived from a method initially employed for epistemic uncertainty quantification in turbulence models (Gorlé et al. [16]). A marker function $m$ is then introduced and it computes the local deviation from a parallel shear flow. This is accomplished by tanking into account the velocity gradient and the streamline at a certain point. According to Gorlé et al. [16], if one takes into consideration a local velocity vector $U_{i}$ and the corresponding unit vector along the streamline $s_{i}=U_{i} / \sqrt{U_{k} U_{k}}$ (namely the velocity vector over its magnitude), the quantity:

$$
g_{j}=s_{i} \frac{\partial U_{i}}{\partial x_{j}}
$$

expresses the gradient of the streamline-aligned velocity, while the ratio:

$$
f=\frac{\left|g_{j} s_{j}\right|}{\sqrt{g_{k} g_{k}}} \equiv \cos \beta
$$


The quantities $s_{i}, g_{j}$ and $\beta$ are briefly illustrated and explained in Figure 2 .

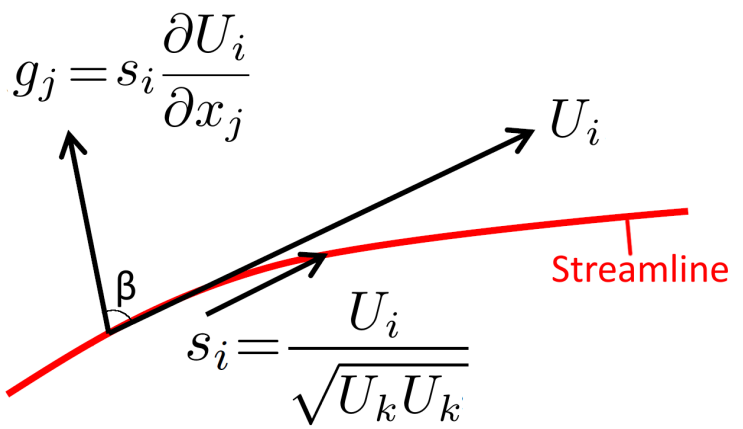
between the gradient of the velocity projected onto the streamline and the direction of the streamline. Consequently, $f$ will be equal to zero in the case of parallel shear flows. A further step could be the definition of this marker also in bulk or free-stream flows, scaling $f$ by the square of the local turbulence intensity:

$$
m=f \frac{k}{U_{k} U_{k}} .
$$

Figure 2: Schematic and visual explanation of $s_{i}, g_{j}$ and $\beta$. Adapted from [18

231 On the basis of this formulation, three marker definitions are employed to

(where $\sqrt{g_{k} g_{k}}$ is the magnitude of $g_{k}$ ) represents the cosine of the angle

define the deviation $\delta$ in a specific range of values, and benchmarked for the detection of the BIA:

- Version 1: $m_{1}=m=f \frac{k}{U_{k} U_{k}}$,

- Version 2: $m_{2}=\left|g_{j} s_{j}\right|$,

- Version 3: $m_{3}=f=\frac{\left|g_{j} s_{j}\right|}{\sqrt{g_{k} g_{k}}}$.

These formulations, together with the deviation ones, contribute in defining the Building Influence Area multifariously, maximising the user's freedom of choice. 


\subsection{Transition formulations}

Once the deviation metric is selected, two formulations for the transition between the different flow regions (namely disturbed and undisturbed) are then available:

1. Polynomial

$$
\phi=\delta^{\alpha} \phi_{\text {wake }}+\left(1-\delta^{\alpha}\right) \phi_{A B L}=\phi_{\text {wake }}+\left(1-\delta^{\alpha}\right)\left(\phi_{A B L}-\phi_{\text {wake }}\right)
$$

\section{Sinusoidal}

$$
\phi=\phi_{\text {wake }}+\left(\phi_{A B L}-\phi_{\text {wake }}\right)\left[1-0.5\left(1+\sin \left(\delta^{*}\right)\right)\right]^{\alpha},
$$

$$
\delta^{*}=\pi \max (\delta-0.5,-0.5) .
$$

Balogh and Parente [3] (2015) adopted a sinusoidal transition function similar to the one proposed in eqs. 11 and 12 .

This specific sinusoidal function was initially formulated and discussed by Peralta et al. [28] (2014) and by Balogh [2] (2014). In eqs. 10, 11 and $12 \delta$ (relative deviation of the actual local $A B L$ quantity, with respect to the inlet one) and $\delta^{*}$ (varying between $-\frac{\pi}{2}$ and $\frac{\pi}{2}$ ) are the blending parameters. $\phi$ is the model parameter to be modified (namely $C_{\mu}, S_{\epsilon}, S_{k}$ and the source terms of the momentum eq.) while $\phi_{\text {wake }}$ and $\phi_{A B L}$ are the values of $\phi$ in the wake and $A B L$ regions respectively.

The parameter $\alpha$ regulates the rapidity/balance of the transition between the undisturbed and disturbed formulations. This is briefly explained in Figure 3 , where, for an increasing value of $\alpha$, the sinusoidal blending results in a sharper detection of the BIA, while the same sharpening effect is obtained for the polynomial transition once $\alpha$ is decreased. 


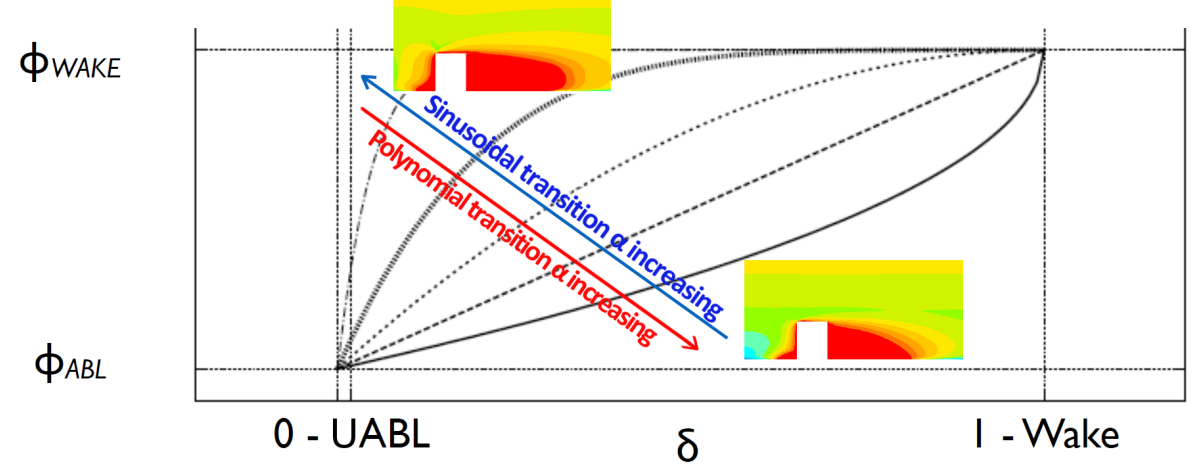

Figure 3: Influence of the $\alpha$ parameter on the blending transition: higher values of $\alpha$ lead to a more disturbed (wake) approach for the sinusoidal blending (blue line), while higher values of $\alpha$ lead to a more undisturbed (UABL) approach for the polynomial blending (red line).

To better explain the behaviour and the effectiveness of the different blending methodologies proposed as well as the spatial distributions of the so-obtained BIA, Figure 4 shows the contour plots of $\delta$ values around a bluff body in the symmetry plane for pure, hybrid and marker blending formulations.

From Figure 4, where $\delta$ is ranging between 0 (blue tone) and 1 (red tone), the Pure $u$ blending formulation is capable of detecting the disturbed flow-field upwind and especially in the wake of the building. Its detecting effect in the downwind region decreases with the improving of the distance from the obstacle. The Hybrid blending (b) results in a sharper and more extended detection of the disturbed area in proximity of the building in respect to the Pure blending formulation (a). This is especially true upwind, over the roof and in the wake of the obstacle and it is due to the fruitful coupling of the two pure deviations $\delta_{u}$ and $\delta_{k}$ which lead to different detection areas.

As for the marker function, displayed in the contour plot (c) from Figure 4, it is evident that its detection is definitely already abrupt around the obstacle, suggesting that a proper tuning of $\alpha$ for this blending formulation would play a minor rule. The detection of the Building Influence Area is further improved in 


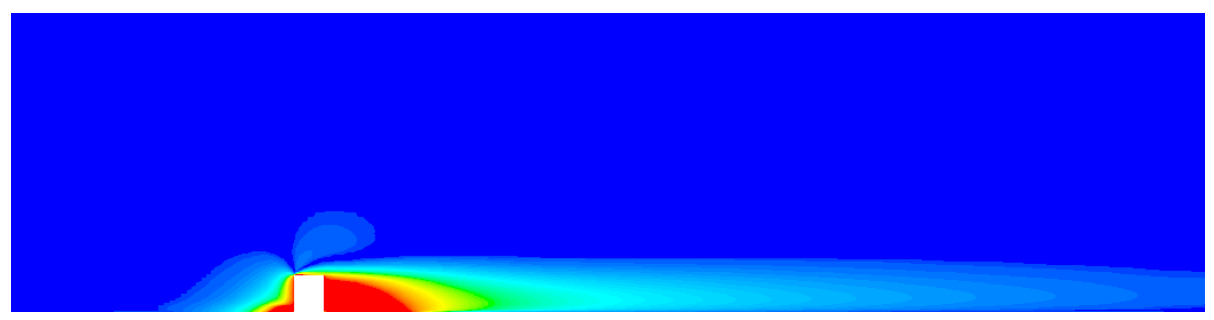

(a) Pure $u$ blending

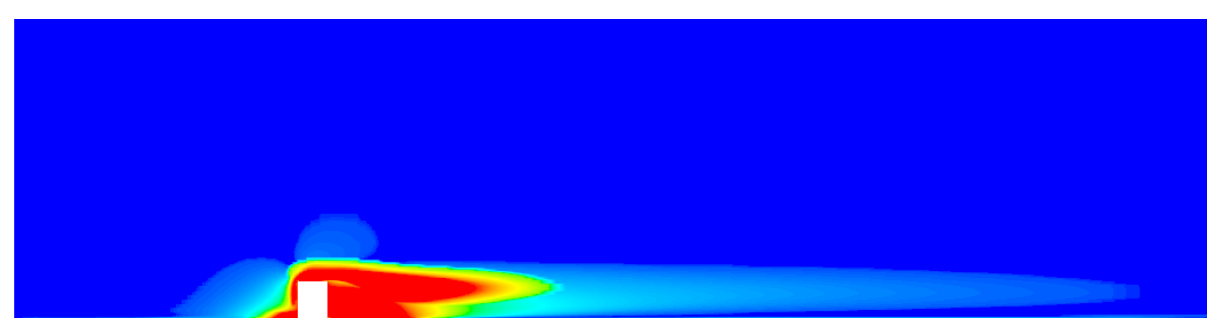

(b) Hybrid blending

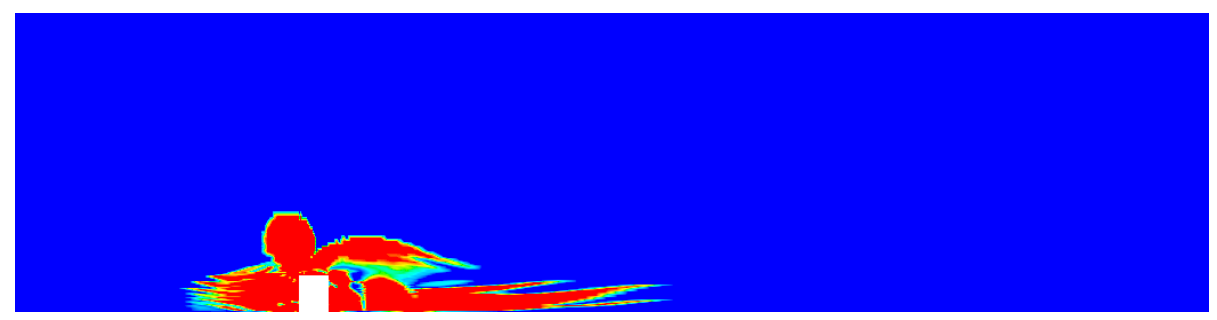

(c) Marker function v.3

Figure 4: Contour plots of the $\delta$ values around a ground-mounted obstacle in the symmetry plane for the pure formulation based on $u$ relative deviation (a), the hybrid one based on both $u$ and $k$ relative deviations (b) and the marker v.3 formula (c)

the upwind region and over the building, while its overall extension downwind is reduced in respect to the deviation formulations (a)-(b).

Focusing the $\alpha$ coefficient, its effect on the $\phi$ parameters and, more in general, on the sharpness and rapidity of the transition can be witnessed displaying in Figure 5 the contour plots of $C_{\mu}$ (being $C_{\mu}$ one of the parameters blended between the comprehensive formulation and the NLEV one) for a pure sinusoidal blending simulation with $\alpha$ equal to 1,4 and 32 respectively. As expected, the area where the NLEV $C_{\mu}$ is applied, improves with the increase of the $\alpha$ coefficient. 
(a) Sinusoidal blending based on velocity deviation, $\alpha=1$

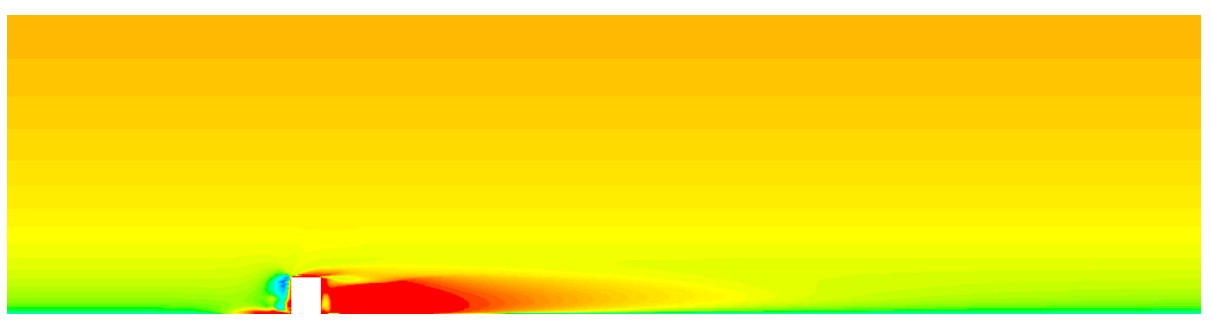

(b) Sinusoidal blending based on velocity deviation, $\alpha=4$

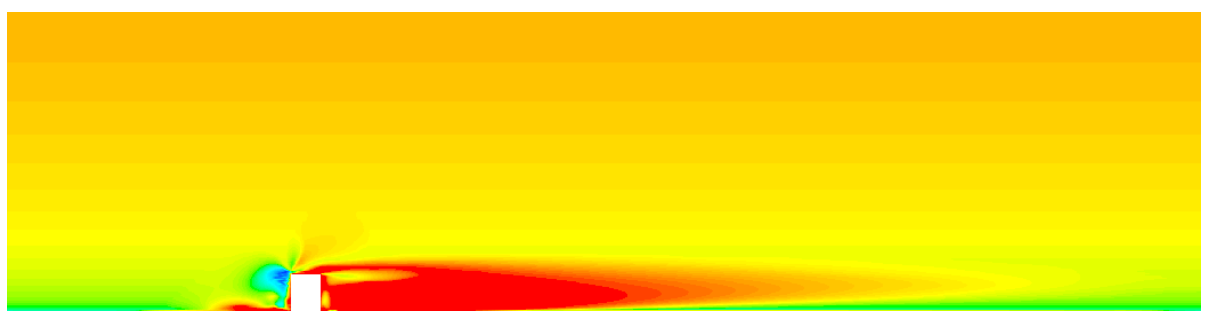

(c) Sinusoidal blending based on velocity deviation, $\alpha=32$

Figure 5: Contour plots of $C_{\mu}$ (Craft NLEV model) around a ground-mounted obstacle in the symmetry plane for a blending formulation based on $u$ relative deviation, setting $\alpha$ to 1 (a) , 4 (b) and $32(\mathrm{c})$

287 Its maximum extension is then reached when $\alpha=32$.

As for the models applied inside the BIA, since the comprehensive approach doesn't assure reliable predictions in disturbed flow-field, the previous investigations (Parente et al. [25], Gorlé [17] ) applied the standard k- $\epsilon$ model in the vicinity of obstacles. This choice proved to be more reliable than the comprehensive approach but still far away from providing satisfactory results. 


\subsection{Non-Linear Eddy-Viscosity models}

Inside the BIA, as an alternative to linear closures, non-linear eddy-viscosity models (NLEV) can be implemented to gain better predictions in disturbed regions. Non-linear models consist in keeping the Kolmogorov-Prantdl eq. and extending the Boussinesq hypothesis to higher order terms. Including all the available cubic terms in the mean velocity gradients, it is possible to deduce the following cubic stress-strain relation, valid for all the non-linear eddy-viscosity models:

$$
\begin{aligned}
& \overline{u_{i}^{\prime} u_{j}^{\prime}}=-2 \nu_{t} S_{i j}+\frac{2}{3} k \delta_{i j}+C_{1} \nu_{t} \frac{k}{\epsilon}\left(S_{i k} S_{j k}-\frac{1}{3} S_{k l} S_{k l} \delta_{i j}\right)+C_{2} \nu_{t} \frac{k}{\epsilon}\left(\Omega_{i k} S_{k j}+\right. \\
& \left.+\Omega_{j k} S_{k i}\right)+C_{3} \nu_{t} \frac{k}{\epsilon}\left(\Omega_{i k} \Omega_{j k}-\frac{1}{3} \Omega_{l k} \Omega_{l k} \delta_{i j}\right)+C_{4} \nu_{t} \frac{k^{2}}{\epsilon^{2}}\left(S_{k i} \Omega_{l j}+S_{k j} \Omega_{l i}\right) S_{k l}+ \\
& +C_{5} \nu_{t} \frac{k^{2}}{\epsilon^{2}}\left(\Omega_{i l} \Omega_{l m} S_{m j}+S_{i l} \Omega_{l m} \Omega_{m j}-\frac{2}{3} S_{l m} \Omega_{m n} \Omega_{n l} \delta_{i j}\right)+ \\
& +C_{6} \nu_{t} \frac{k^{2}}{\epsilon^{2}} S_{i j} S_{k l} S_{k l}+C_{7} \nu_{t} \frac{k^{2}}{\epsilon^{2}} S_{i j} \Omega_{k l} \Omega_{k l}
\end{aligned}
$$

where $S_{i j}$ and $\Omega_{i j}$ are the strain-rate tensor and the vorticity tensor components. The inclusion of cubic terms leads to a model which can show sensitivity to streamline curvature and swirl (Craft et al. [9]). The various NLEV models differ from each other by the definition they give to $C_{\mu}$ and by the values they attribute to the different coefficients $C_{i}$ appearing in the recursive eq. 13. Suga optimized the coefficients $C_{1}$ to $C_{7}$ over a range of flows (see Craft et al. [9], 1996), deducing the following set of model coefficients:

$C_{1}=-0.1 ; C_{2}=0.1 ; C_{3}=0.26 ; C_{4}=-10 C_{\mu}^{2} ; C_{5}=0 ; C_{6}=-5 C_{\mu}^{2} ;$

$C_{7}=5 C_{\mu}^{2}$

where $C_{\mu}$, according to Craft [9], is given by:

$$
C_{\mu}=\min \left(0.09, \frac{1.2}{1+3.5 \eta}\right)
$$


311

312

313

314

According to the Lien formulation [21] 1996, $C_{\mu}$ and the $C_{1}-C_{7}$ coefficients are defined as follows:

$C_{1}=\frac{0.75 / C_{\mu}}{1000+S^{3}} ; C_{2}=\frac{3.75 / C_{\mu}}{1000+S^{3}} ; C_{3}=\frac{4.75 / C_{\mu}}{1000+S^{3}} ; C_{4}=-10 C_{\mu}^{2} ; C_{5}=0 ;$

$C_{6}=-2 C_{\mu}^{2} ; C_{7}=2 C_{\mu}^{2}$,

where $C_{\mu}$ is defined as:

$$
C_{\mu}=\frac{2 / 3}{1.25+S+0.9 \Omega}
$$

The last cubic formulation considered is the one proposed by Ehrhard [11] in 2000 , as an improvement of the previous NLEV models:

$C_{1}=-0.05 ; C_{2}=0.1 ; C_{3}=0.5-\frac{1}{4} \exp \left(-(S-\Omega)^{2}\right) ; C_{4}=-4 C_{\mu}^{2} ; C_{5}=0 ;$ $C_{6}=-2 C_{\mu}^{2} ; C_{7}=2 C_{\mu}^{2}$,

where $C_{\mu}$, is given by:

$$
C_{\mu}=\min \left(0.15, \frac{1}{0.9 S^{1.4}+0.4 \Omega^{1.4}+3.5}\right) .
$$

\subsection{Wall treatment}

Considering the relevance of the surface roughness and the high Reynolds numbers typically associated with $A B L$ flows, the adoption of wall functions is generally required for near-wall modelling.

The standard smooth law of the wall has the following form:

$$
\frac{U_{p}}{u_{*}}=\frac{\ln \left(y^{+}\right)}{\kappa}+B
$$

with the integration constant $B=5.0-5.4$. The effect of roughness is then modelled through the introduction of a shift in the intercept, $\Delta B\left(k_{S}^{+}\right)$. Thus, 
the following expression is obtained for the logarithmic rough law of the wall:

$$
\frac{U_{p}}{u_{*}}=\frac{1}{\kappa} \ln \left(E y^{+}\right)-\Delta B\left(k_{S}^{+}\right),
$$

with $E$ being the wall function constant $(E=9-9.7935)$. The function $\Delta B\left(k_{S}^{+}\right)$ depends on the dimensionless roughness height, $k_{S}^{+}=\frac{u_{*} k_{S}}{\nu}$. When $k_{S}^{+}>90$ :

$$
\Delta B\left(k_{S}^{+}\right)=\frac{1}{\kappa} \ln \left(C_{S} k_{S}^{+}\right),
$$

which gives:

$$
\frac{U_{p}}{u_{*}}=\frac{1}{\kappa} \ln \left(\frac{E y^{+}}{C_{S} k_{S}^{+}}\right)
$$

32 where $C_{s}$ is a roughness constant. By comparing Equations (1) and 20, it becomes evident that the two treatments are inconsistent, leading to discrepancies in the prediction of the near wall velocity. Therefore, a proper selection of the roughness constants has to be performed. In this regard, Blocken et al. [6] proposed a first order match between the velocity inlet profile and the rough law of the wall in correspondence of the first cell centroid, $z_{p}$, with the aim of performing an appropriate selection of $C_{S}$ :

$$
\frac{E y^{+}}{C_{S} k_{S}^{+}}=\frac{z_{p}+z_{0}}{z_{0}} \rightarrow C_{S}=\frac{E \frac{u_{*} z_{p}}{\nu} z_{0}}{\frac{u_{*} k_{S}}{\nu}\left(z_{0}+z_{p}\right)} \sim \frac{E z_{0}}{k_{S}} \sim \frac{E z_{0}}{z_{p}} .
$$

In Equation [21, a common requirement of $A B L$ simulations is then made explicit: the distance $z_{p}$ between the centroid of the wall-adjacent cell and the wall has to be greater than the sand-equivalent roughness $k_{s}$ of the terrain.

This requirement can be translated into an upper limit for $k_{s}$, giving: $z_{p} \geq k_{S}$ ([6]). However, even when the value of the velocity at the first cell matches the one provided by Equation 11, the standard rough wall function suffers from two main drawbacks. First, it is constrained by the maximum size of the wall 
adjacent cell. In fact, at the first cell centroid, $z_{p}$, Equations 20 gives:

$$
\frac{U_{p}}{u_{*}}=\frac{1}{\kappa} \ln \left(\frac{E}{C_{S}}\right)
$$

347 taking $k_{S}=z_{p}$. This implies that $C_{S}$ cannot be greater than the value of the parameter $E$. Furthermore, the standard wall function does not imply any direct effect of the roughness properties on the turbulence quantities at the wall.

Parente et al. [24, 26, 27] proposed an implementation of the rough wall function preserving the form of the universal law of the wall, through the introduction of a new wall function constant and non-dimensional wall distance:

$$
\frac{U_{p}}{u_{*}}=\frac{1}{\kappa} \ln \left(\widetilde{E} \widetilde{y}^{+}\right)
$$

with

$$
\widetilde{y}^{+}=\frac{u_{*}\left(z+z_{0}\right)}{\nu} \quad \widetilde{E}=\frac{\nu}{z_{0} u_{*}},
$$

with the non-dimensional distance, $\widetilde{y}^{+}$, being a $y^{+}$shifted by the aerodynamic roughness, and the new wall function constant, $\widetilde{E}$ depending on the roughness characteristics of the surface. In Equation (22) the friction velocity $u_{*}$ is computed locally as $u_{*}=C_{\mu}^{0.25} k^{0.5}$. This approach removes the aforementioned drawbacks of the standard wall function, without constraining its flexibility [26, 27]. More specifically, its extension to mixed rough and smooth surface is achieved through a simple redefinition of the law of the wall constants. Furthermore, it enables an extended flexibility from the point of view of mesh generation, considering that the wall function parameters do not impose any specific limitation on the first cell height [26]. 


\section{Computational method and boundary conditions}

The present Section introduces the test-cases adopted for the computational analysis, namely the Cedval $\mathrm{A} 1-1$ and $\mathrm{B} 1-1$ test cases from the BLASIUS Wind Tunnel of the Environmental Wind Tunnel Laboratory of the Meteorological Institute of Hamburg University [43]. Computational modelling of the $\mathrm{ABL}$, both for the ground-mounted building and the cluster of building configurations, is carried out using ANSYS Fluent 17. The results were obtained setting the steady, $3 d$, double precision, pressure based solver. The standard discretization scheme was applied to pressure, while second order schemes were adopted for momentum and turbulence quantities, and the SIMPLE algorithm was selected for pressure-velocity coupling.

\subsection{Cedval A1-1 single building}

The first test case is the Cedval A1-1, displaying a single building. As shown in Figures 1 and 6 , the building has length $L=0.1 \mathrm{~m}$, width $W=0.15 \mathrm{~m}$, height $H=0.125 \mathrm{~m}$ and 4 source elements on the leeward building side (suitable for a dispersion study). The origin of the coordinate system is located at the center of the bottom face of the building, with the $z$-axis pointing upwards and the $x$-axis pointing downstream. The computational domain inlet boundary is set $1 \mathrm{~m}$ upstream of the centroid of the building (corresponding to the upwind area covered by a smooth floor in the wind tunnel [38]) whereas the outlet boundary is located $3 m$ downstream of the origin of the coordinate system (satisfying the requirement of a distance larger than $10 \mathrm{H}$ between the building and the outflow boundary [38]). Considering the symmetry of the model with respect to the plane $y=0 m$, only half of the domain has been studied, through a mirror function. The width and height of the so-obtained domain are $0.75 \mathrm{~m}$ and $1 \mathrm{~m}$ respectively, corresponding to the wind tunnel size. A structured mesh consist- 


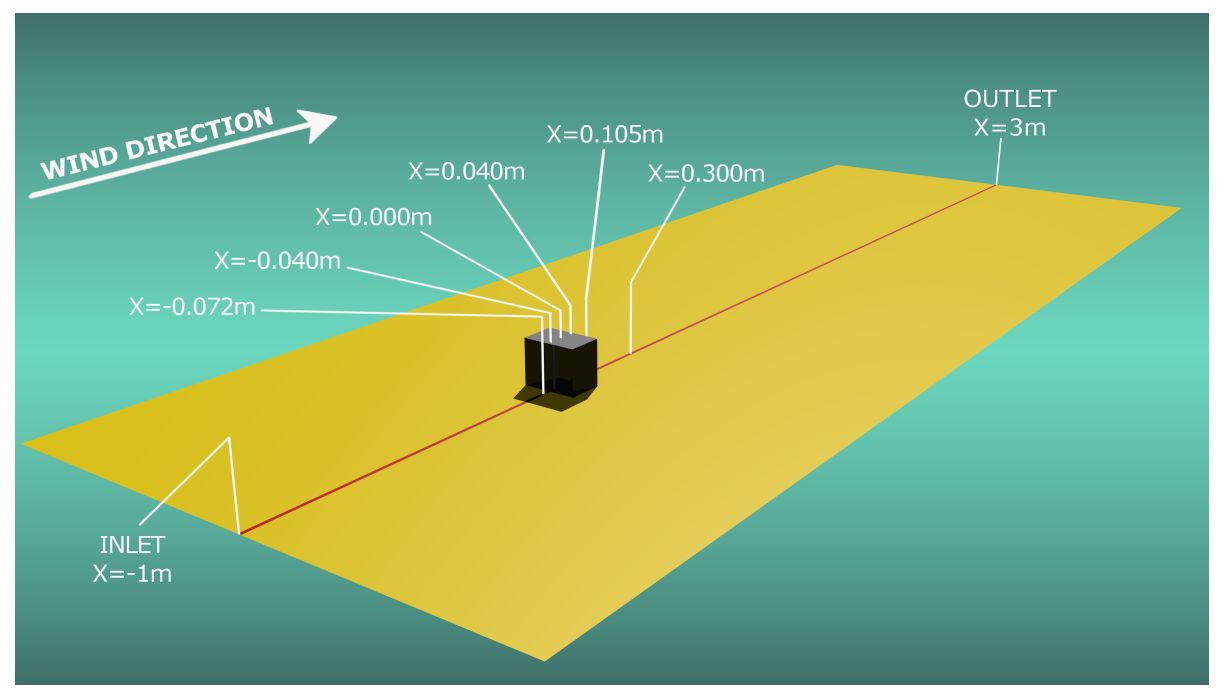

Figure 6: Cedval A1-1 geometry view, including the measurement lines taken into consideration. The red line indicates the intersection of the ground with the symmetry plane $(y=0 m)$, where a mirror function is applied for reproducing the other half of the domain.

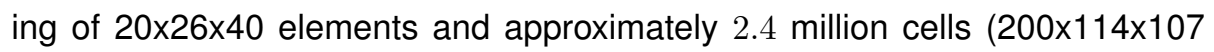
elements) is adopted. The height of the ground adjacent cell is $0.00075 \mathrm{~m}$. According to the AIJ guidelines proposed by Tominaga et al. [38], the lateral and top boundaries of the computational domain extend more than $5 \mathrm{H}$ (5.5H for the lateral edges and $7 \mathrm{H}$ for the top edge, with $H$ being the height of the target obstacle) from the external edges of the ground mounted building. Moreover, the building blockage ratio is equal to $1.25 \%$, not exceeding, as a consequence, the recommended value (3\%) [38]. In regions with a steep velocity gradient, the stretching ratio of adjacent grids has been set to 1.3, according once again to AlJ [38]. COST advises the same limitation for grid stretching ratio (Franke et al. [14]). A grid independence study had already been carried out by Parente et al. [26] for the same mesh in order to estimate the solution error associated to the discretization selected. According to this test, two supplementary grids were generated, whose coarsening ratio was $r_{h}=h_{i} / h_{1}=1.15$ (Roache [34]) in the three directions and with an overall number of cells of about 1.74 
and 1.26 millions. Referring to COST Action 732 (Franke et al. [13]), error estimates were computed for turbulent kinetic energy and velocity, through a proper comparison between the results provided by the three grids proposed (wherever experimental data were available). Focusing on the solution uncertainty, $U_{\text {sver }}$, Parente et al. [26] followed the guidelines proposed by Logan and Nitta [22], while the grid convergence index ( $\mathrm{GCl}$ ) was determined assuming a safety factor, $F_{s}=1.25$ (Roache [34]). For the finest grid, a maximum grid convergence index $(\mathrm{GCl})$ of $5 \%$ and $3 \%$ was determined for velocity and turbulent kinetic energy respectively, by averaging the values obtained at all the measurement locations [26]. As for the boundary conditions definition, the building sides and top, as well as the tunnel sides have been set as stationary smooth walls, while the tunnel ground was modelled as rough wall. The entrance of the domain is defined as velocity inlet, imposing the turbulence profiles from Table 1. while the end of the domain is specified as pressure outlet imposing $k$ and $\epsilon$ profiles as backflow. Finally the tunnel top is set as velocity inlet, imposing the inlet velocity profile for computing the longitudinal component of velocity at the top height and specifying, also, the $k$ and $\epsilon$ profiles.

\subsection{Cedval B1-1 Array of Building}

The simulation of the flow around an array of obstacles is considered in order to further validate the proposed approaches. As shown in Figure 7, it displays an array of $3 \times 7$ buildings, having the same dimensions of the single building in the A1-1 test case. The center of the bottom face of the blue building is the origin of the Cartesian coordinate system. $x, y$ and $z$ directions are identical to the ones adopted in the one building case (namely $x$ oriented in the wind direction and $z$ in the upwards direction). The computational domain inlet boundary is set $1 \mathrm{~m}$ upstream of the first array of building (namely $1.85 \mathrm{~m}$ upstream of the co- 


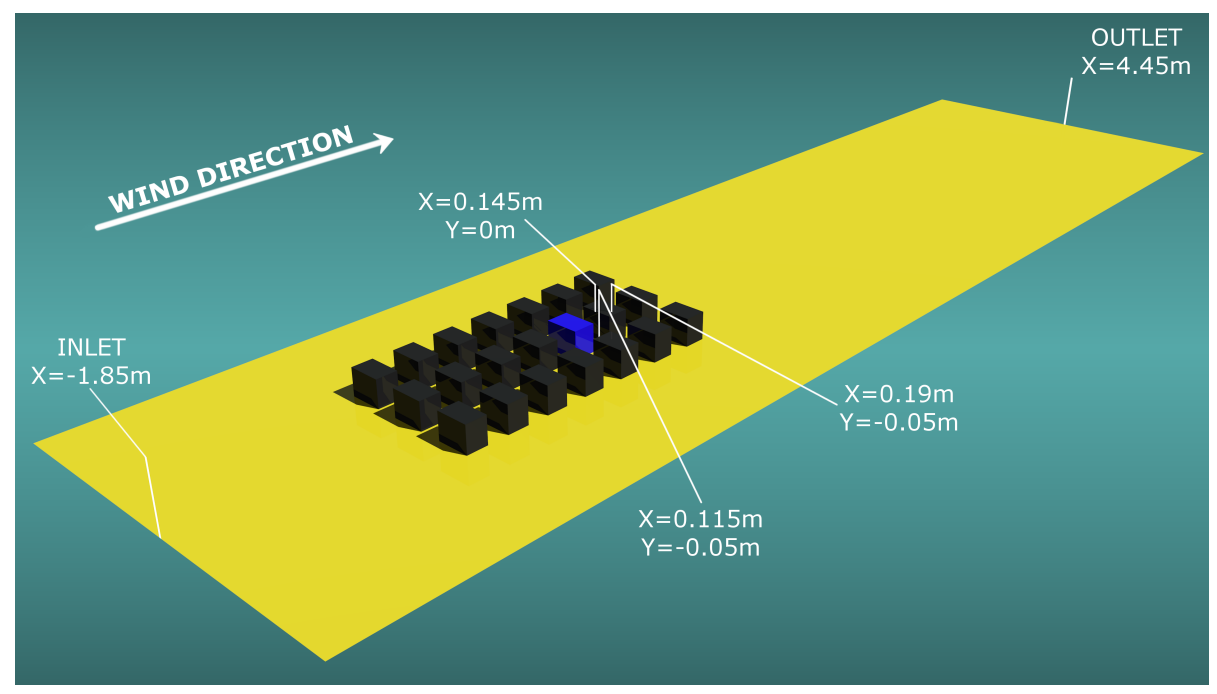

Figure 7: Cedval B1-1 geometry view, including the measurement lines taken into consideration.

ordinate center), where $A B L$ profiles are measured in the wind tunnel, whereas the outlet boundary is located $4 m$ downstream of the last array of building. The width and height of the domain are $1.5 \mathrm{~m}$ and $1 \mathrm{~m}$ respectively, corresponding to the wind tunnel size. Also for the B1-1 test case, in regions of interest, the stretching ratio of adjacent grids has been set to 1.3. Both for Cedval A1-1 and B1-1 test cases, hexahedral meshes have been selected and generated, in order to achieve a correct representation of the building aerodynamics. In fact, avoiding tetrahedral and pyramid cells leads to a better convergence with second order schemes [7]. Analogously to the A1-1 test case, a grid sensitivity analysis was carried out with the aim of quantifying the solution error associated to the discretization grade. The fine mesh consists of approximately 3.5 millions of cells and one coarser mesh has been generated with 2.3 millions of cells (coarsening ratio $r_{h}=1.17$ ). When comparing two meshes instead of one, the guide lines advises a more conservative safety factor, namely $F_{S}=3$ [34]. Such a higher factor of safety is recommended for reporting purposes and is conservative of the actual errors. For the finest grid, $\mathrm{GCl}$ of $2 \%$ and $2 \%$ 
was determined for $u$ and $k$ respectively. The boundary conditions have been specified as equally done for the A1-1 single building (section 3.1), with the only main difference that the domain has been entirely represented, without the implementation of a mirror function in the symmetry plane.

\section{Results}

The present Section shows the performances of the proposed models, namely different blending configurations, for the simulation of the $A B L$ around a single building and an array of buildings, in combination with various NLEV closures.

\subsection{Cedval A1-1 Single Building}

Figures 8 and 9 show the $u$ and $k$ measurements taken from eight different distances along the $x$ axes in the symmetry plane. The first set of models tested includes the standard $k-\epsilon$ (orange line) and the comprehensive approach jointed to pure sinusoidal blending with Craft NLEV model applied inside the BIA and $\alpha$ tuned to 1, 4 and 32 (the green, blue and black dashed lines respectively). For the std $k-\epsilon$ model the $S_{\epsilon}$ source term has been added to the $\epsilon$ transport equation in order to make the inlet conditions analytical solutions of the $k$ and $\epsilon$ transport equations. As for the choice of the blending transition, no major differences have been observed between the application of a sinusoidal or a polynomial formulation in terms of performance, but the transition selection is mostly related to a stability matter. As a consequence, the choice will fall on the formulation that guarantees the highest stability and the lowest scalar residuals. From Figures 8 , the $k-\epsilon$ model fails in correctly reproducing the velocity field. First of all, it is not able to preserve the inlet profile from the rise of horizontal inhomogeneity. This is evident once the comparison between 


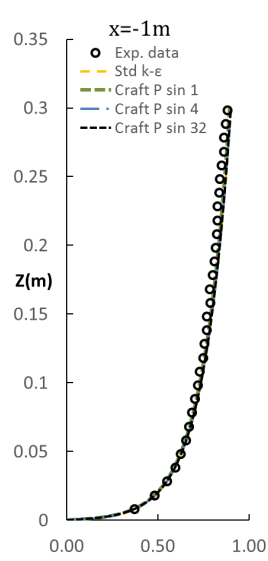

(a)

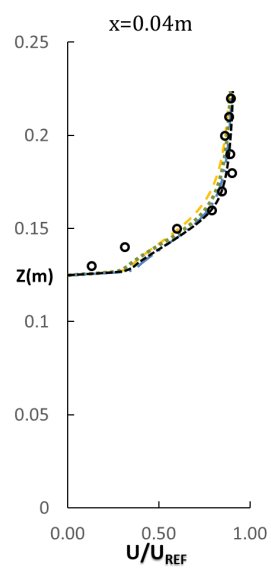

(e)

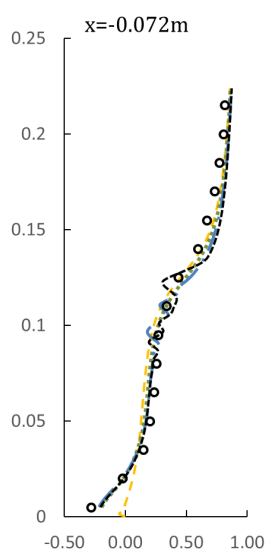

(b)

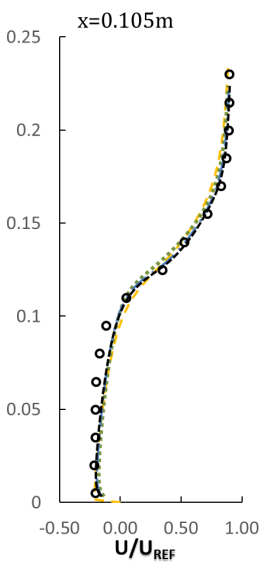

(f)

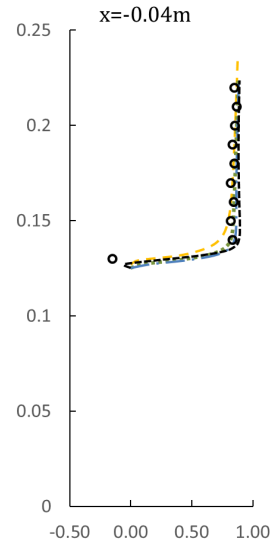

(c)

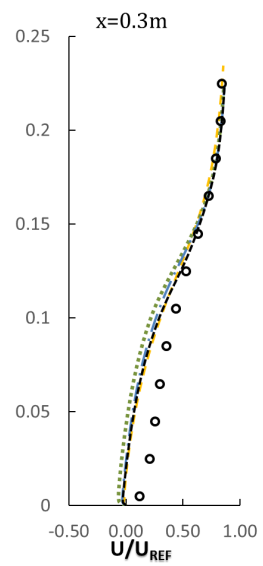

(g)

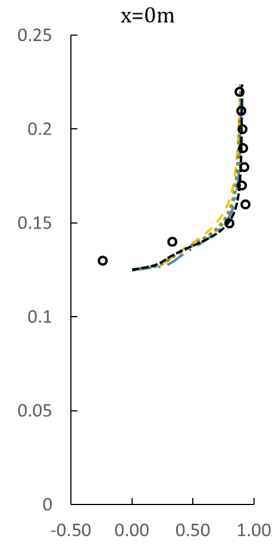

(d)

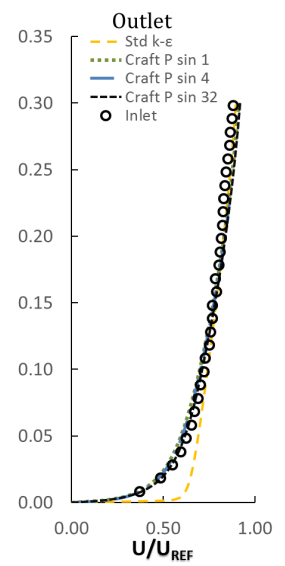

(h)

Figure 8: Comparison of experimental and numerical predictions of non-dimensional velocity at different axial locations (a-g) and at the outlet section of the domain (h), using the standard $k-\epsilon$ model and Craft closure for the wake in sinusoidal blending with $\alpha=1,4,32$.

472 the inlet profile and the outlet one is taken into consideration in Figure 8 (h).

${ }_{473}$ This is a well known problem of CFD simulations of $A B L$ flows, intrinsically re-

474 lated to the selection of proper boundary conditions. The undesired changes

475 (streamwise gradients or horizontal inhomogeneity) occur in the vertical pro-

476 files of mean wind speed and turbulence quantities as they travel from the inlet

477 of the computational domain, even in open field, down to the outlet. This phe- 


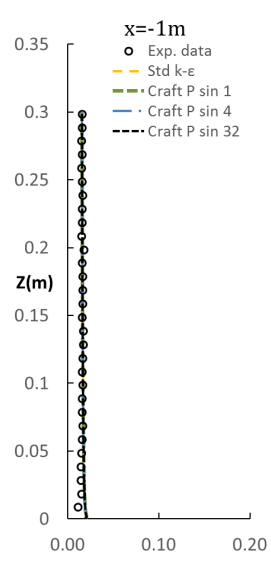

(a)

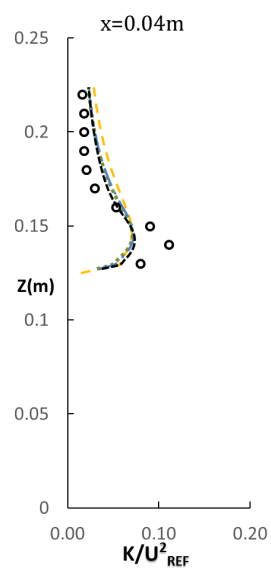

(e)

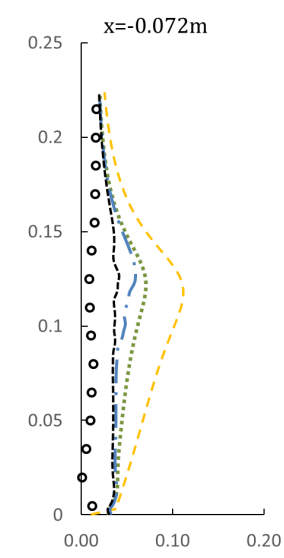

(b)

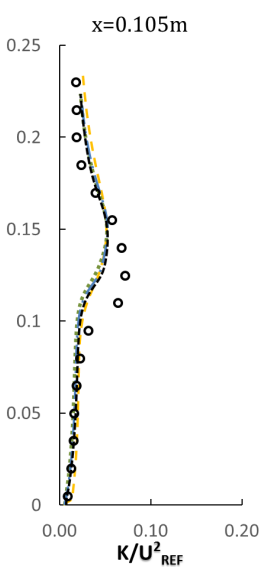

(f)

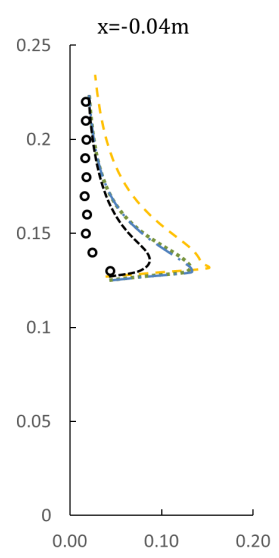

(c)

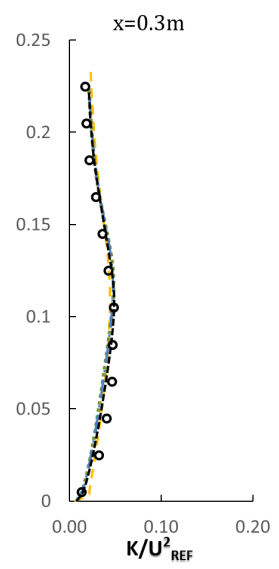

(g)

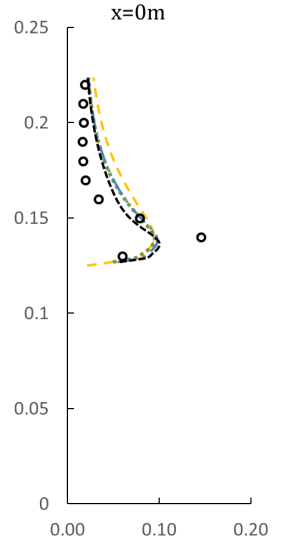

(d)

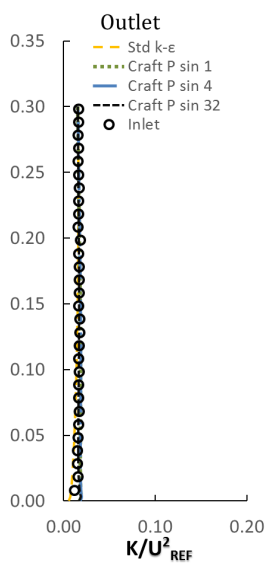

(h)

Figure 9: Comparison of experimental and numerical predictions of non-dimensional turbulent kinetic energy at different axial locations (a-g) and at the outlet section of the domain (h), using the standard $k-\epsilon$ model and Craft closure for the wake in sinusoidal blending with $\alpha=1,4,32$.

nomenon has been described in detail by Blocken et al. [5], leading to a severe worsening of the quality and validity of the simulation results. As stated by Pontiggia et al. [29], the reason for these horizontal variations in the profiles lies in a progressive rise of turbulence intensity in the vicinity of the ground produced by the terrain roughness together with a quick disappearance of turbulent intensity once moving away from ground level (due to the lack of shear stress in 
the flat-profile air flow). This, in its turn, can be translated in an incompatibility of the inlet profiles with the applied wall functions, their roughness parameters, the computational grid and the turbulence model. Furthermore, the std $k-\epsilon$ misrepresents the recirculation zones next to the wall and the building, e.g. the non-dimensional velocity profile in correspondence of $x=-0.072 m$ in Figure 8 (b). Here the blending configurations starring Craft closure behave more accurately than the std $k-\epsilon$ even if slightly showing instability at $z=0.1-0.15 m$ (especially for $\alpha=32$ ). This behaviour is related to this specific NLEV model and will not be witnessed with the application of the NLEV models proposed by Lien and Ehrhard.

Even greater improvements are registered once focusing on the non-dimensional turbulent kinetic energy profiles, shown in Figure 9. The standard $k-\epsilon$ model misrepresents (namely over-predicts) turbulent kinetic energy values almost everywhere in the vicinity of the obstacle and, in particular, in the upwind recirculation zone (b), over the obstacle - mostly in the impinging side of the building (c) - and in the downwind stagnation region ( $\mathrm{f}-\mathrm{g})$. The over-prediction of $k$ is reduced once the blending approach is applied and especially when the $\alpha$ parameter is tuned to high values, indicating a very fast and more extended transition from the homogeneous to the disturbed flow-field, where the nonlinear approach is applied. This trend confirms that a blending transition from an undisturbed formulation to the disturbed one should be fast and effective. Keeping the comprehensive approach outside the BIA, the very next step is to test the other NLEV models (namely the ones proposed by Lien and Ehrhard) against the Craft closure. For this simulation, the best blending configuration is then kept, namely the one with $\alpha=32$, using a pure blending and a sinusoidal transition. The outcomes are shown in Figures 10 and 11. All the non-linear eddy-viscosity models contribute in a better reproduction of both velocity and 


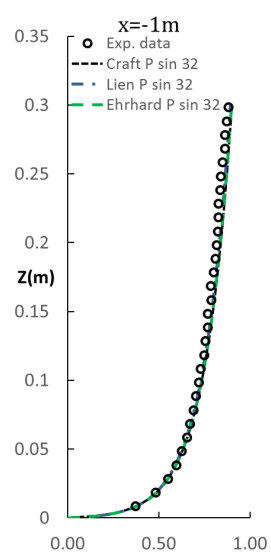

(a)

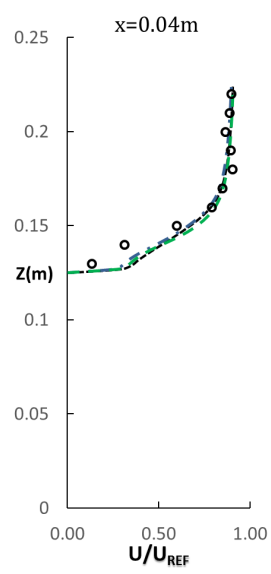

(e)

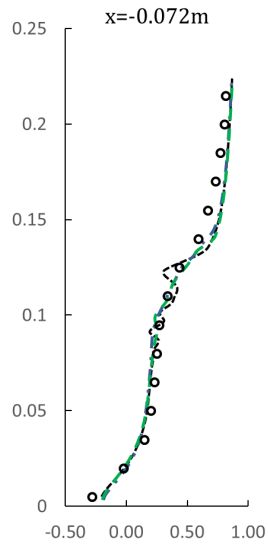

(b)



(f)



(c)

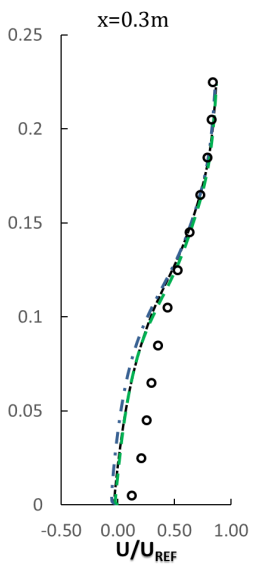

(g)

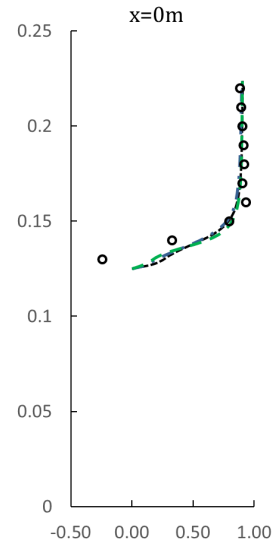

(d)

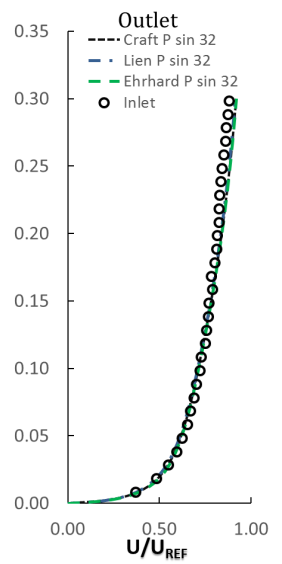

(h)

Figure 10: Comparison of experimental and numerical predictions of non-dimensional velocity at different axial locations (a-g) and at the outlet section of the domain (h), using Craft, Lien and Ehrhard NLEV models for the wake in sinusoidal blending with $\alpha=32$.

${ }_{511}$ turbulent kinetic energy fields with respect to the standard $k-\epsilon$ model. As for non-dimensional velocity, no substantial differences are noticed. The only significant improvement is witnessed at $x=-0.072 \mathrm{~m}$, with both Lien and Ehrhard trending away from Craft behaviour and thus preventing the profile from the occurrence of abrupt velocity variations. For all the other heights, the three model predictions become comparable, almost collapsing onto one single line. 




(a)



(e)

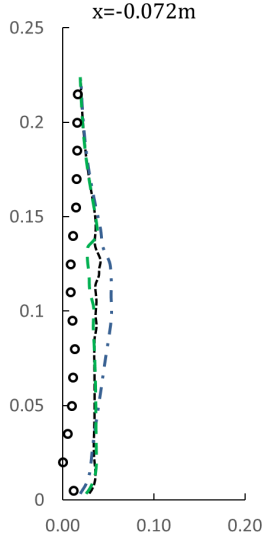

(b)

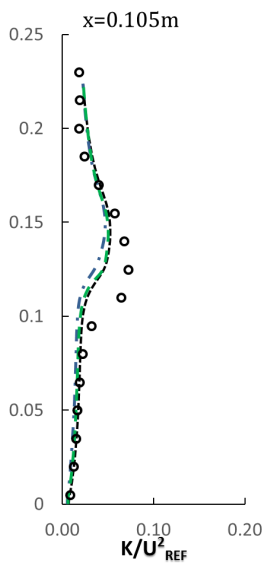

(f)

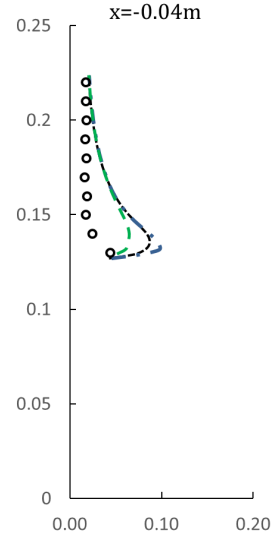

(c)

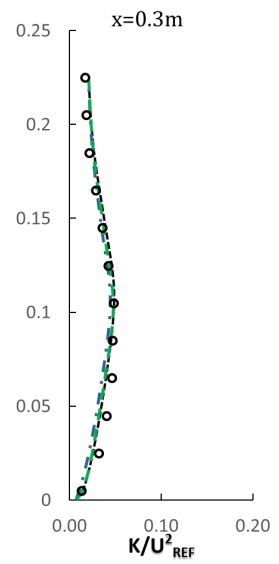

(g)

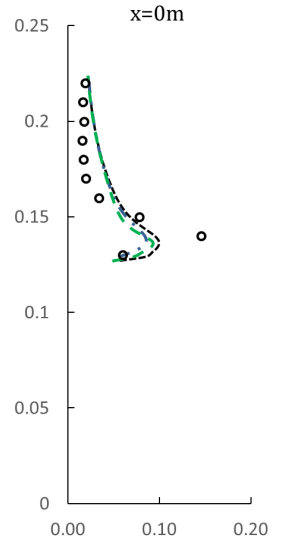

(d)

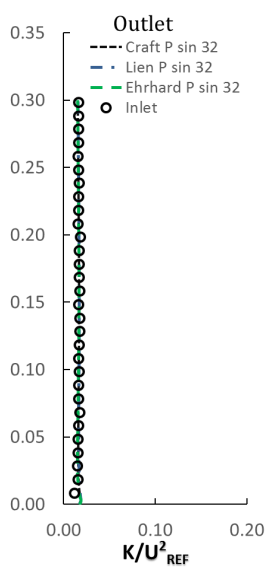

(h)

Figure 11: Comparison of experimental and numerical predictions of non-dimensional turbulent kinetic energy at different axial locations (a-g) and at the outlet section of the domain (h), using Craft, Lien and Ehrhard NLEV models for the wake in sinusoidal blending with $\alpha=32$.

517 In particular Craft, Lien and Ehrhard approaches show a light over-prediction of velocity next to the roof of the building (c-d) and slightly underestimate $u$ ${ }_{519}$ at $x=0.3 m(\mathrm{~g})$ from Figure 10 in the wake. But it is with $k$ profiles in Figure 11 that the greatest differences between the NLEV models are witnessed.

521 Ehrhard provides the best results and further reduces the over-prediction of $k$.

${ }_{522}$ The subsequent simulation for the single building A1-1 test case aims at inves- 


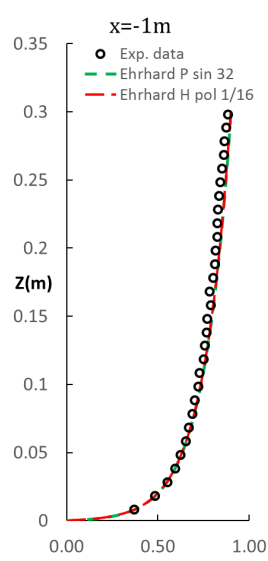

(a)

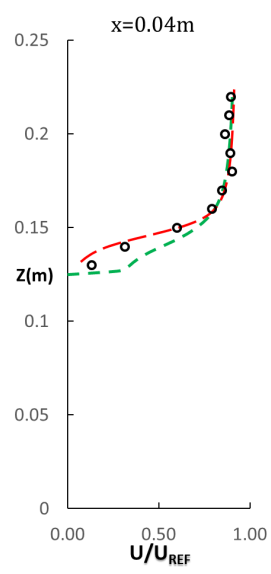

(e)

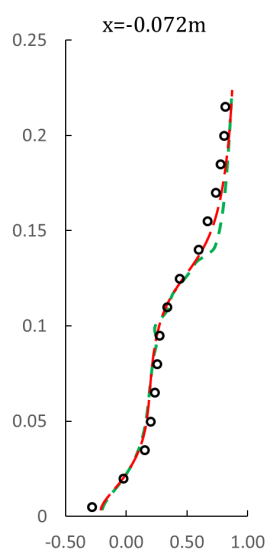

(b)

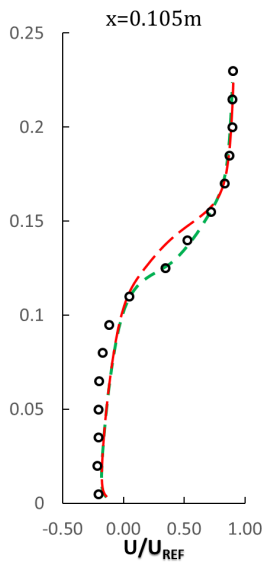

(f)

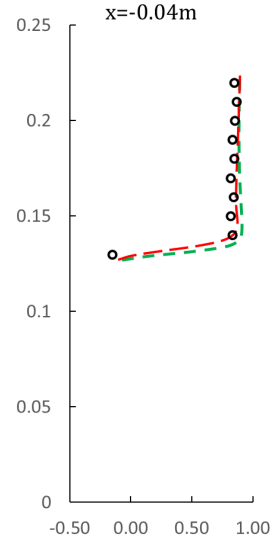

(c)

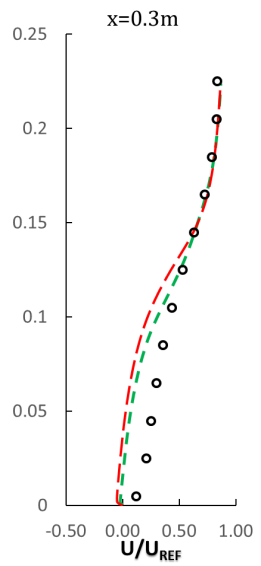

(g)

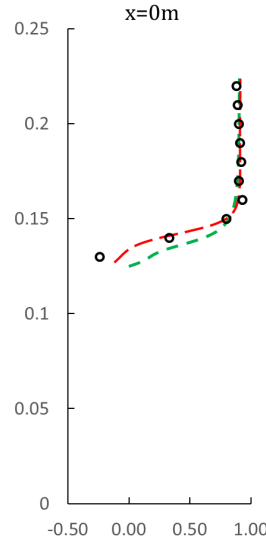

(d)



(h)

Figure 12: Comparison of experimental and numerical predictions of non-dimensional velocity at different axial locations (a-g) and at the outlet section of the domain (h), using Ehrhard in sinusoidal pure blending with $\alpha=32$ and Ehrhard in polynomial hybrid blending with $\alpha=1 / 16$ for the wake.

${ }_{523}$ tigating the role of the blending formulation on the $u$ and $k$ predictions. To this

${ }_{524}$ purpose the simulation outcomes obtained using Ehrhard model (with $\alpha=32$

${ }_{525}$ and sinusoidal transition), using pure blending (based on $u$ ), are benchmarked

526 with the results provided by the Ehrhard model (with $\alpha=1 / 16$ ) using hybrid

${ }_{527}$ blending (based on $u$ and $k$ ). The profiles of this simulation are shown in Fig528 ures 12 and 13 . From $u / U_{R E F}$ profiles it is evident that the application of 


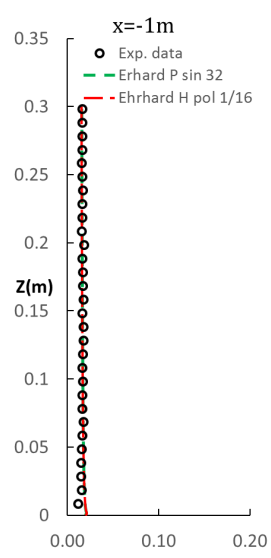

(a)



(e)

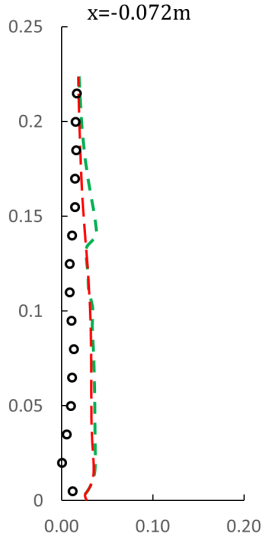

(b)

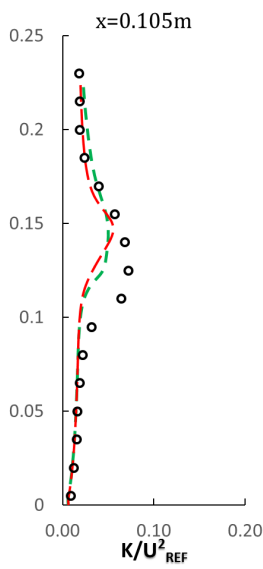

(f)

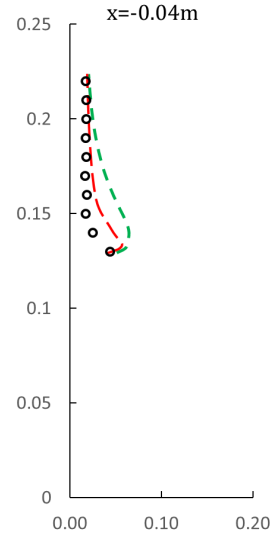

(c)

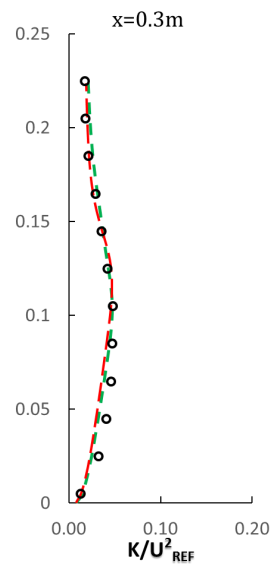

(g)



(d)

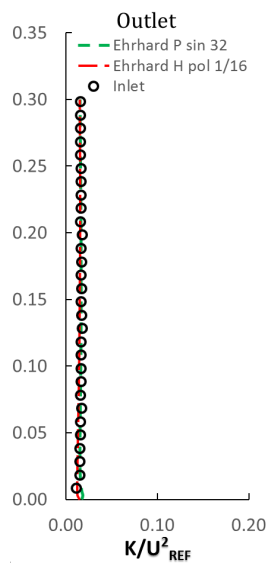

(h)

Figure 13: Comparison of experimental and numerical predictions of non-dimensional turbulent kinetic energy at different axial locations $(\mathrm{a}-\mathrm{g})$ and at the outlet section of the domain $(\mathrm{h})$, using Ehrhard in sinusoidal pure blending with $\alpha=32$ and Ehrhard in polynomial hybrid blending with $\alpha=1 / 16$ for the wake.

529 a hybrid blending, rather than a pure one, results in an improved prediction 530 of experimental data, with a more accurate representation of the recirculation ${ }_{531}$ zones. As for non-dimensional turbulent kinetic energy, its over-prediction is 532 further reduced when applying the faster and more extended transition guaran-

${ }_{533}$ teed by the hybrid blending. This is true especially in the upwind recirculation ${ }_{534}$ area $-x=-0.072 m$ - and in the leading edge $-x=-0.04 m$ - in Figure 13 . 


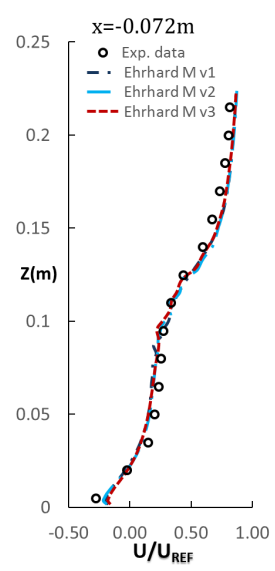

(a)



(e)

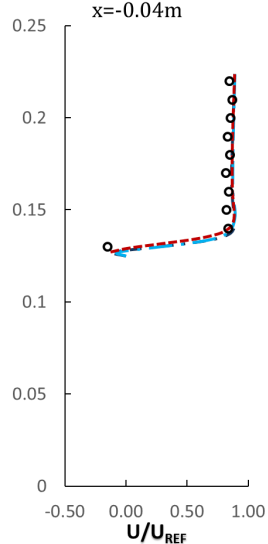

(b)

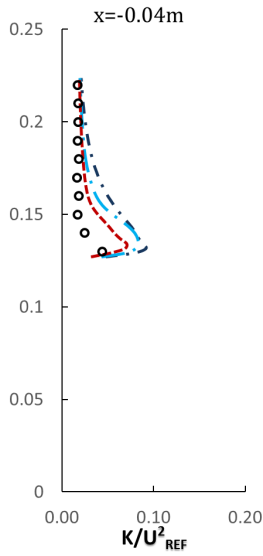

(f)



(c)



(g)

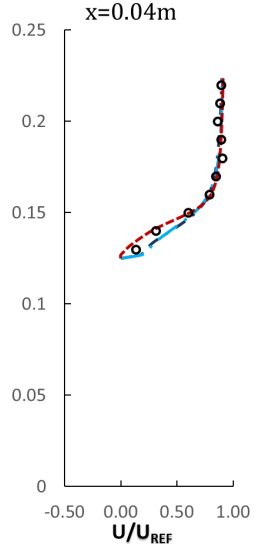

(d)

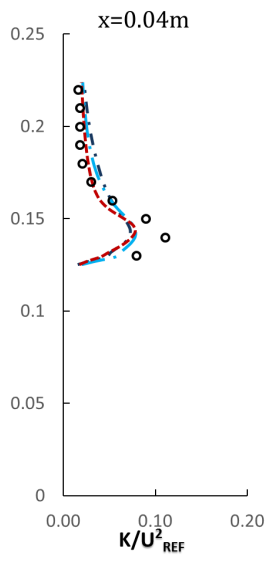

(h)

Figure 14: Comparison of experimental and numerical predictions of non-dimensional velocity and turbulent kinetic energy at different axial locations of the domain, using three different marker definitions and Ehrhard NLEV model in sinusoidal transition for the wake.

As for the BIA detection based on the marker functions, simulations are first carried out to compare the different marker performances. All the markers have been intensively tested both in polynomial and sinusoidal transition, with all the three NLEV models and varying $\alpha$.

To sum up the outcomes of this benchmark, only four representative heights in the symmetry plane are taken into account (upwind the building with $x=$ 
${ }_{541}-0.072 m$ and on top of the building with $x=-0.04 m, x=0 m, x=0.04 m$ ) for $u$

${ }_{542}$ and $k$. The results are shown in Figure 14.

${ }_{543}$ All the marker approaches proved to be able to represent both the velocity field

${ }_{544}$ (especially in the recirculation areas) and turbulent kinetic energy field (in par-

${ }_{545}$ ticular in the stagnation zones) with remarkable accuracy. Once again, as for

${ }_{546}$ stability and performance, no significant differences were observed between

${ }_{547}$ the results obtained using the sinusoidal or the polynomial transition.

${ }_{548}$ Moreover, the parameter $\alpha$ has a minor effect on the results. This indicates that

549 the use of the marker results in a sharp definition of the region of application

550 of NLEV model, without the need for tuning this specific blending parameter.

${ }_{551}$ The results shown in Figure 14 for the different markers are obtained using a

552 sinusoidal blending transition and $\alpha=1$.

${ }_{553}$ From these profiles, it can be observed that, above all the markers, v.3 (cosine

${ }_{554}$ of the angle between the direction of the streamline and the gradient of the

555 velocity projected onto the streamline) is capable of getting the best results for velocity over the building (b-d), in particular at the center of the roof $(x=0 \mathrm{~m})$. But it is especially in the prediction of $k$, (e-h) from Figure 14 , that marker v.3

558

outmatches the alternatives, leading to a general reduced over-prediction of $k$, especially in the impinging side of the building, as illustrated in Figure 14 (f).

One possible physical explanation for this performing behaviour, could be intrinsically linked to the formulation of this marker. In fact, the cosine of this angle is a measure that, by definition and not being scaled by any local turbulence intensity, shows a great sensitivity to fluid disturbance, catching efficiently both smooth and abrupt variations.

The subsequent profiles displayed in Figure 15 for the A1-1 test case are meant to compare the best blending configuration based on the Hybrid blending and the best blending transition based on the marker formulation (marker v.3). Sec- 


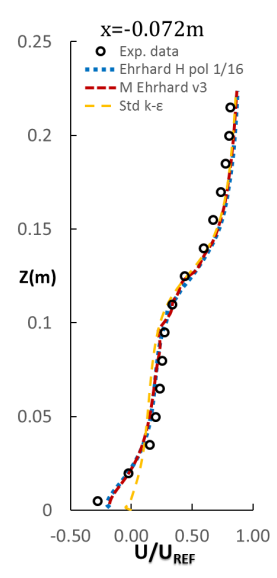

(a)

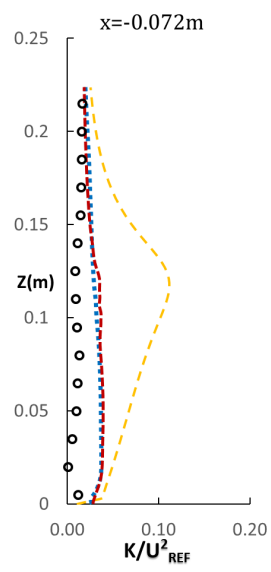

(e)

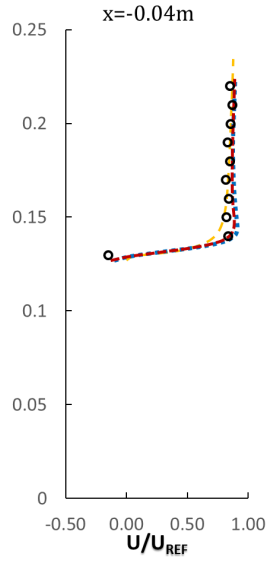

(b)

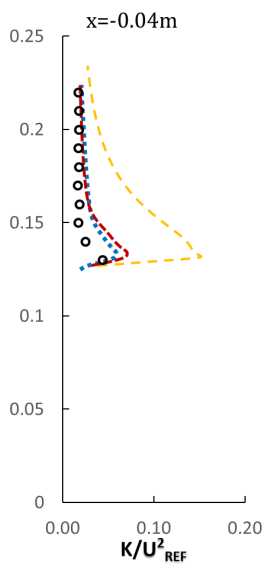

(f)

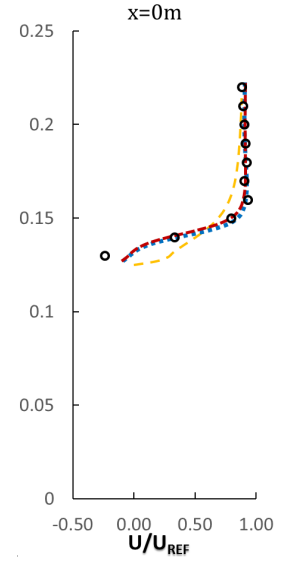

(c)



(g)

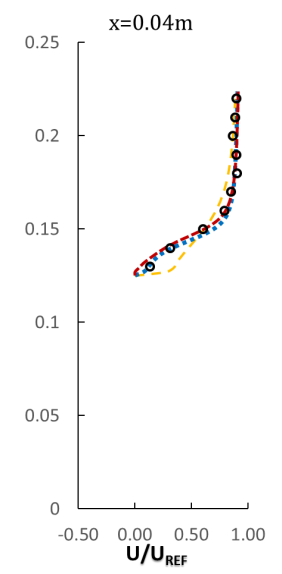

(d)

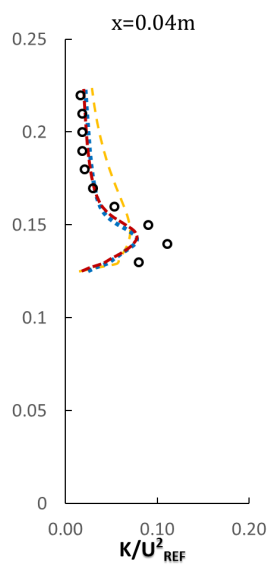

(h)

Figure 15: Comparison of experimental and numerical predictions of non-dimensional velocity and turbulent kinetic energy at different axial locations of the domain, using both Hybrid configuration and marker v. 3 with Ehrhard NLEV model for the wake against the standard $k-\epsilon$ model.

571

572 backs which can be spotted from the turbulence profiles.

${ }_{573}$ The first one is related to the over-prediction of turbulent kinetic energy in 
the upwind recirculation zone and in the impinging side of the building $(x=$ $-0.072 m$ and $x=-0.04 m)$. In these locations, even if improving the overall performance in respect to the standard $k-\epsilon$ model, the most performing approaches (hybrid blending and marker formulation v.3) still slightly over-predicts turbulent kinetic energy. This behaviour is only partly related to the effectiveness and accuracy of the Building Influence Area, whose setting parameters have been tuned to the highest values. But it is mostly explainable as an intrinsic limitations of NLEV models formulations and, more in general, of steady RANS simulations. In support of this argument, different simulations testing the NLEV approaches extended to all the domain have been run, resulting in outcomes comparable/equal (namely the same over-prediction of $k$ ) to the the hybrid blending and marker ones. A similar consideration can also be given for the velocity field at $x=0.3 \mathrm{~m}$, in the wake. At this location, all the methodologies applied show an evident under-prediction of velocity in the near-ground region (namely from $z=0 m$ to $z=0.13 m$ ).

Since this behaviour was also witnessed when extending NLEV models to all the domain and considering that this zone is completely detected by the BIA (Figure 4), the explanation lies once again in an intrinsic limitation of steady RANS models in accurately representing the velocity field in every region of the wake.

With the aim of providing a more qualitative comparison of the performance of the blending approaches, Figures 16 and 17 display the contour plots of velocity magnitude and turbulent kinetic energy for the standard $k-\epsilon$ model, Craft NLEV in pure sinusoidal blending with $\alpha=1,4,32$, Ehrhard NLEV in hybrid polynomial blending with $\alpha=1 / 16$ and finally Ehrhard NLEV coupled with marker v.3 formulation. As for velocity magnitude in Figure 16, among all the approaches compared in the contour plots, the standard $k-\epsilon$ model displays 


\section{$\begin{array}{lllllllll}0 & 0.78 & 1.56 & 2.34 & 3.12 & 3.90 & 4.86 & 5.46 & 6.50\end{array}$}



(a)

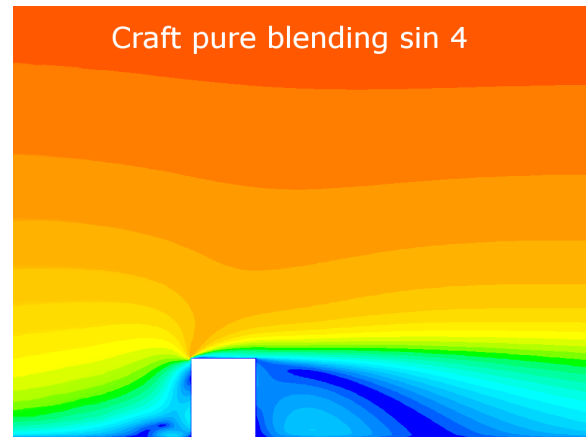

(c)

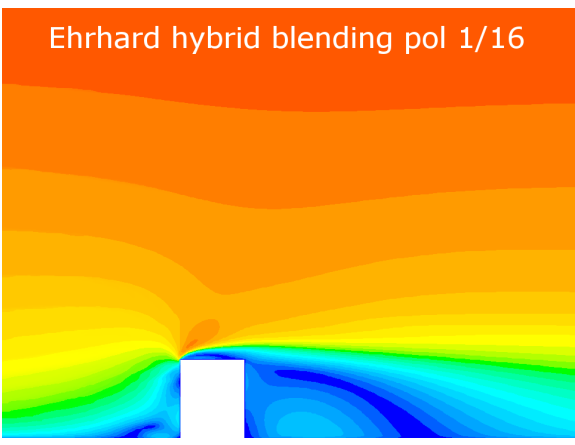

(e)

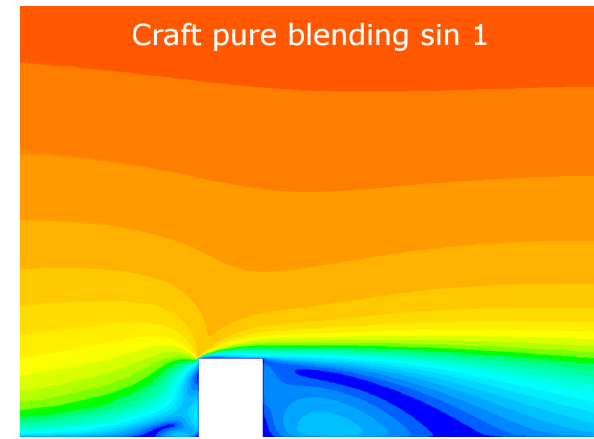

(b)



(d)

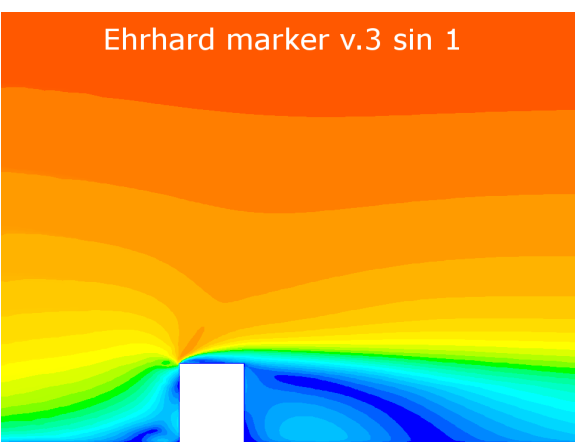

(f)

Figure 16: Contour plots of velocity magnitude for the Cedval A1-1 test case on the plane of symmetry $(y=0 m)$ for standard $k-\epsilon$ model, Craft NLEV in sinusoidal pure blending and $\alpha=1,4$, 32 , Ehrhard NLEV coupled with hybrid blending and marker function v. 3 


\section{$\begin{array}{lllllllll}0 & 0.9 & 1.80 & 2.70 & 3.60 & 4.50 & 5.40 & 6.30 & 7.50\end{array}$}

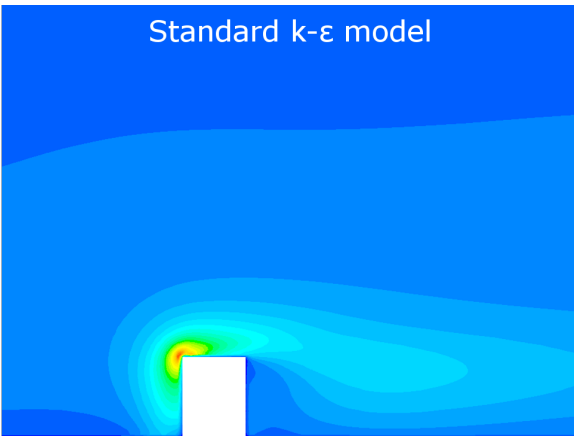

(a)

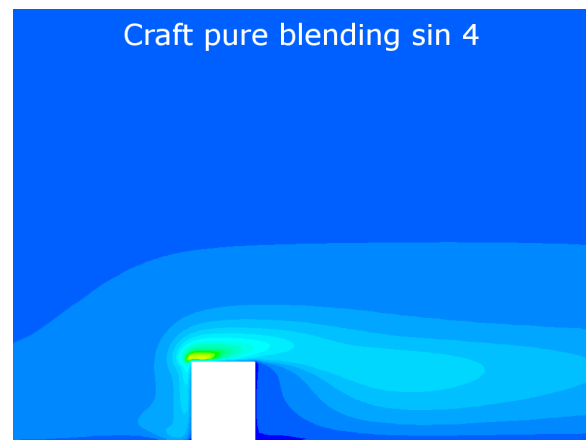

(c)

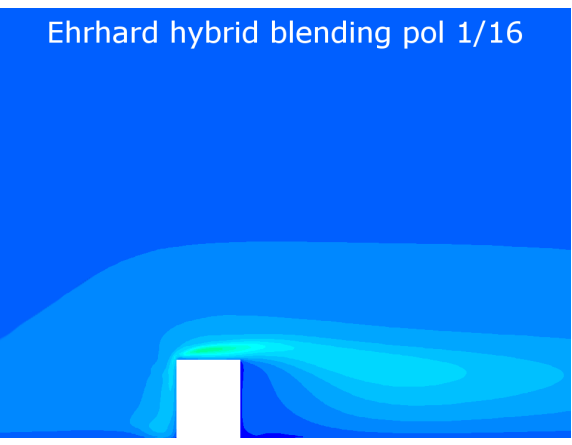

(e)

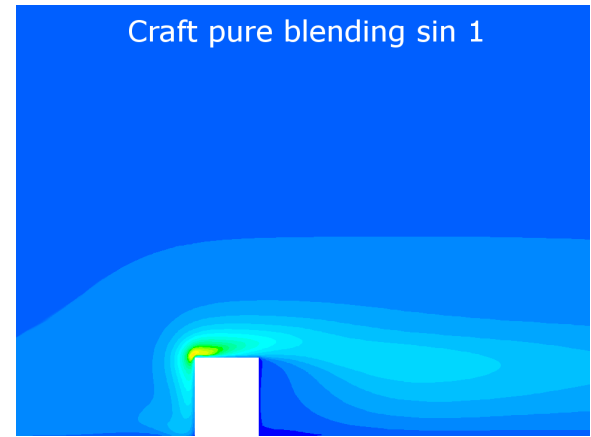

(b)

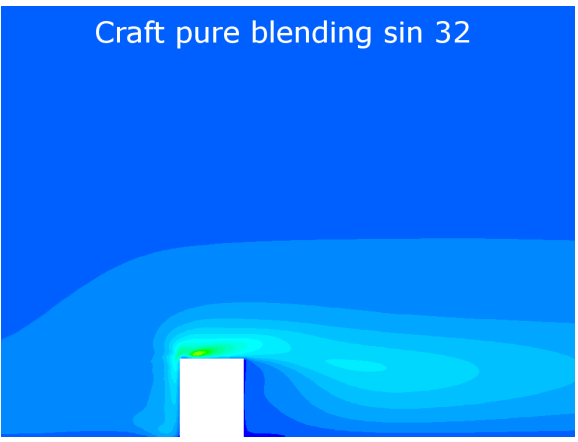

(d)



(f)

Figure 17: Contour plots of turbulent kinetic energy for the Cedval A1-1 test case on the plane of symmetry $(y=0 m)$ for standard $k-\epsilon$ model, Craft NLEV in sinusoidal pure blending and $\alpha=1,4$, 32 , Ehrhard NLEV coupled with hybrid blending and marker function v.3 
the most limited upwind recirculation zone. Focusing on this area, in contrast to what observed in the experimental velocity profile at $x=-0.072 m$ (in the upwind recirculation zone), std $k-\epsilon$ is not capable of detecting any vorticity, thus resulting in a velocity magnitude which is equal (or next) to 0 .

All the other approaches displaying the building influence area concept and NLEV models (b-f from Figure 16), reproduce a slightly more extended wake. Moreover, recirculation zones are now witnessed upwind of the building. Among all the blending approaches, there are not major differences in performance, as also confirmed by the outcomes of velocity profiles.

Still in accordance with velocity profiles, from the same contour plots (b-f), a low-prediction of the velocity values can be observed around a specific area inside the wake (the dark blue area bounded between $x=2 H$ and $x=3 H$ downwind the building 16 .

Focusing on turbulent kinetic energy contours in Figure 17, it is evident that the standard $k-\epsilon$ model over-predicts $k$ in the upwind vorticity area, in the leading edge of the obstacle, over the roof and, finally, in the wake. But this peak of turbulent kinetic energy (especially in the impinging side of the bluff body) is damped and "transported" downwind on the roof of the building, in accordance with the theoretical background and experimental data ( $k$ profiles at these locations), as soon as the automatic BIA with the NLEV models is applied and the $\alpha$ parameter is tuned to high values.

As a consequence, unlike the velocity contours, the turbulent kinetic energy contours highlight significant differences also among the models displaying the BIA. In this regard, it is especially with the Hybrid blending and the marker function v.3 that turbulent kinetic energy overproduction is further limited. In order to extrapolate a more quantitative and immediate information about the performances of the different apporaches tested, the most relevant results 
633

634

643 and corresponding to a parabolic line [19].

${ }_{644}$ The greatest advantage of this statistical evaluation is the possibility to sum up 645 in just two values (namely $M G$ and $V G$ ), a huge amount of experimental data, 646 giving an immediate feedback of the model performance.

${ }_{647}$ Obviously, due to its intrinsic formulation, this specific representation can be a statistical evaluation based on a recommendation by Fox [12], subsequently modified by Hanna [19].

is based on the calculation of the geometric mean bias (MG), geometric variance (VG) and fraction within a factor of two (FAC2):

$$
M G=\exp \left(\overline{\ln \left(P_{e} / P_{m}\right)}\right)
$$

$$
F A C 2=\text { fraction of data for which } 0.5<P_{m} / P_{e}<2
$$

where $P_{e}$ is the experimental property considered and $P_{m}$ is the corresponding modelled property. In an "ideal" model, both MG and VG should be equal to 1.0 .

Geometric mean bias (MG) values equal-under 0.5 and equal-over 2.0 can be thought of as factor of two (FAC2) respectively over-predictions and underpredictions of the geometric mean. The factor of two relation for MG is represented by two vertical lines. The relation - $\ln V G=(\ln M G)^{2}$ - is valid, defining the minimum possible value of geometric variance for a given geometric mean used only for positive properties. Figure 18 displays Geometric mean bias (MG) and Geometric Variance (VG) for the various model applied in the A1-1 test case, referred to non-dimensional turbulent kinetic energy. Also the vertical red lines representing the factor of two, and the parabola of the minimum values

for the non-dimensional turbulent kinetic energy can be summed up through 


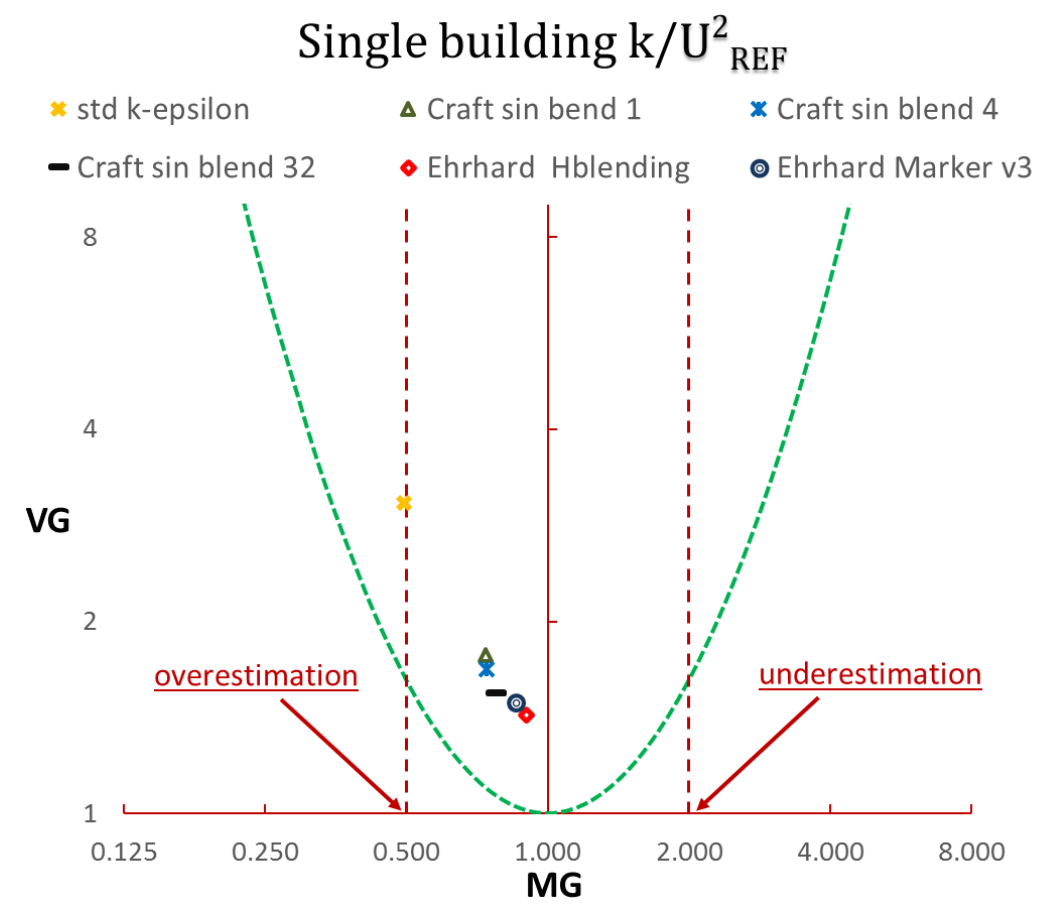

Figure 18: Statistical evaluation of the models performances using the geometric mean BIAS and geometric variance.

of VG for MG are displayed. This "quantitative" evaluation (being a benchmark referred to the mean performance of the model) is in full accordance with the

"qualitative" one (providing more localized informations as expounded by the turbulence profiles) showing that Ehrhard model with hybrid blending and polynomial transition (red rhombus) provides the best predictions, followed very closely by the marker v.3 formulation (blue concentric circles). As expected, the less performing model is the standard $k-\epsilon$, resulting in the most severe over-prediction of $k$.

In addition to the previous simulations, on the basis of the study performed by Merci et al. [23], the two most accurate blending configurations (hybrid blending and marker v.3) have been tested setting the NLEV coefficients of the stressstrain relation 13 equal to zero: $C_{1}-C_{7}=0$. 
As a consequence, the higher order terms of the Reynolds stresses are neglected and only the NLEV $C_{\mu}$ formulation (whose prominence had already been proved by Merci et al. [23]) is kept. From the resulting $u$ an $k$ profiles (which are not shown in this study), it is possible to notice that the results are not worsened but, on the contrary, the so-obtained models perform equally to the "non-zero" NLEV ones (interestingly a further slight limitation of $k$ overprediction is witnessed at $x=-0.072 m)$. As for the accuracy of results, this is clearly not a substantial improvement, but it is made more attractive by the fact that neglecting the higher order terms of the stress-strain relation helps in further limiting computational costs.

Concluding the simulations for the A1-1 single building, a direct comparison between one of the most performing methodologies among those proposed (Ehrhard NLEV model applied in hybrid blending) and (traditionally) more advanced models in respect to the standard $k-\epsilon$ is proposed. The selected approaches for this last test session are the RNG $k-\epsilon$, the Realizable $k-\epsilon$ and the SST $k-\omega$ model.

For the RNG $k-\epsilon$ and the Realizable $k-\epsilon$ model, the same inlet profiles from Table 1 are applied. For the SST $k-\omega$ model, the same profiles for $u$ and $k$ turbulence quantities are applied, while, as for $\omega$ turbulence property, the following profile is adopted [32]:

$$
\omega=\frac{u_{*}}{\sqrt{\beta^{\prime}} \kappa_{k-\omega} z}
$$

The outcomes are displayed in Figures 19 and 20 . Briefly, from the upwind $u$ and $k$ profiles (a-b-c), the less accurate behaviour is the one related to the Realizable $k-\epsilon$ model, which performs similarly to the std $k-\epsilon$. In particular, the SST $k-\omega$ shows the most accurate prediction for $k$ at $x=-0.072 m$, even if still not completely fitting the experimental data.

Focusing on the other locations (over the building and in the wake) the worst 




(a)

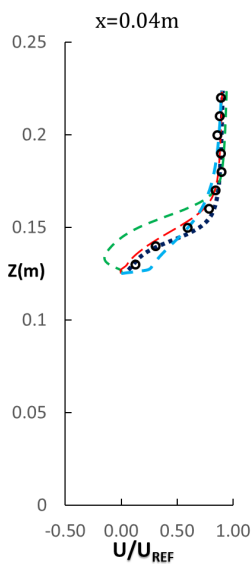

(e)

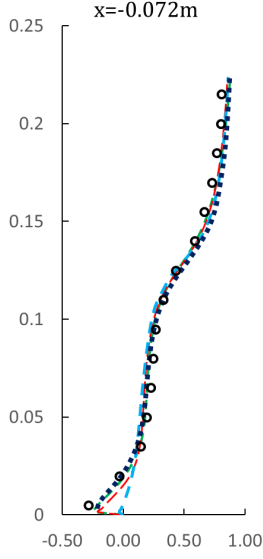

(b)

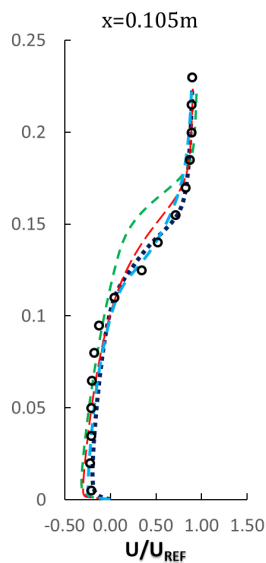

(f)



(c)

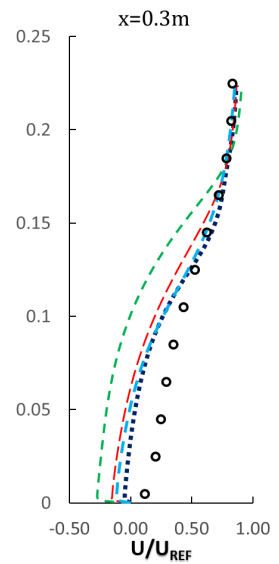

(g)

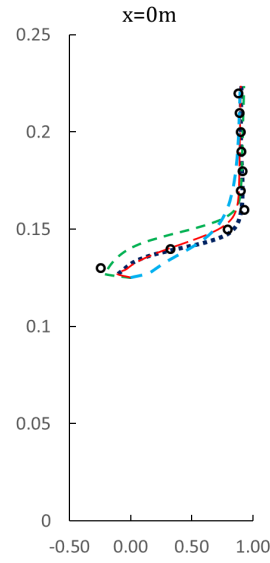

(d)

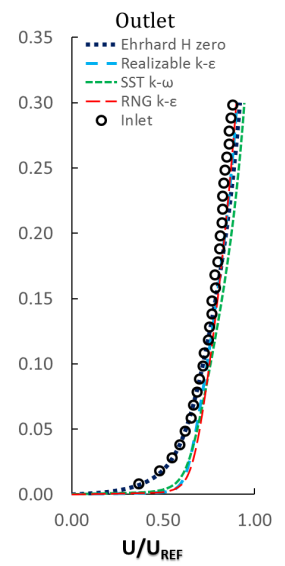

(h)

Figure 19: Comparison of experimental and numerical predictions of non-dimensional velocity at different axial locations (a-g) and at the outlet section of the domain $(\mathrm{h})$, using Realizable $k-\epsilon$, RNG $k-\epsilon$ and SST $k-\omega$ against Ehrhard NLEV model applied inside the BIA in hybrid blending configuration.

performing model, both for $u$ and $k$, is the SST $k-\omega$. Moreover, the Realizable $k-\epsilon$, RNG $k-\epsilon$ and SST $k-\omega$ models are not capable of correctly predicting velocity at $x=0.3 \mathrm{~m}$ (even worsening the predictions of Ehrhard in hybrid blending) and they cannot prevent the rise of horizontal inhomogeneity affecting the profiles. Among the three models, the RNG $k-\epsilon$ shows the most 




(a)

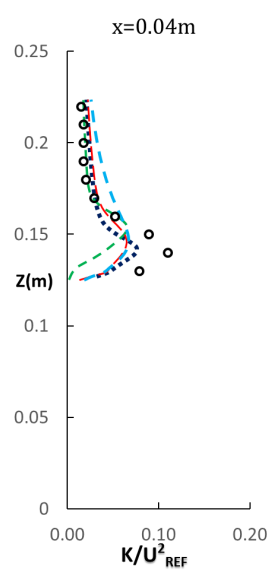

(e)

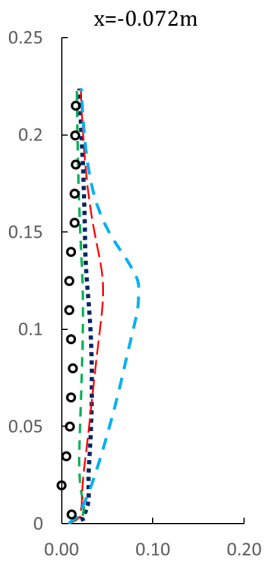

(b)

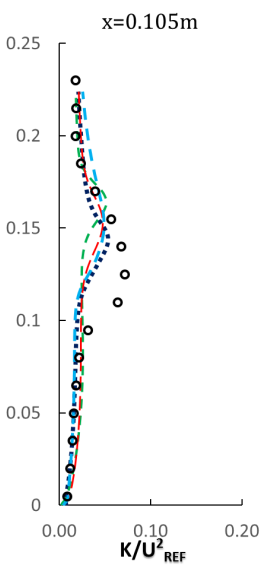

(f)



(c)

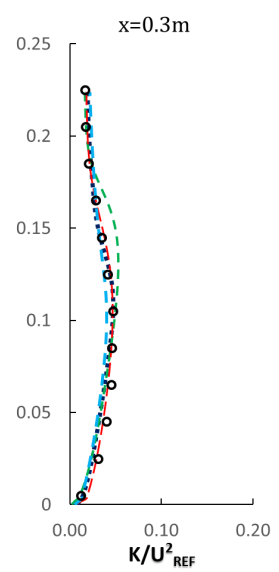

(g)

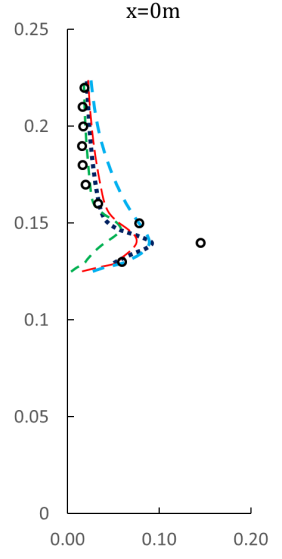

(d)

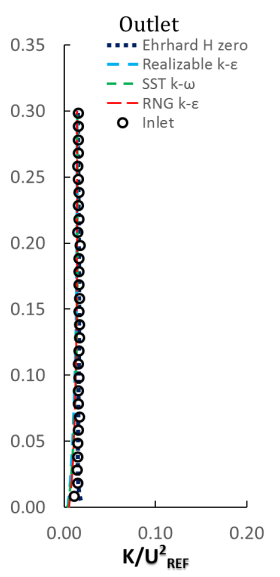

(h)

Figure 20: Comparison of experimental and numerical predictions of non-dimensional turbulent kinetic energy at different axial locations (a-g) and at the outlet section of the domain (h), using Realizable $k-\epsilon$ model, RNG $k-\epsilon$ model and SST $k-\omega$ against Ehrhard NLEV model applied inside the BIA in hybrid blending configuration

competitive performance.

${ }_{696}$ The results obtained in the upwind recirculation zone by the SST $k-\omega$ model

${ }_{697}$ and more in general by the RNG $k-\epsilon$ model, suggest the development of comprehensive approaches also for these models, in order to derive the maximum 699 benefit from their capacities. 


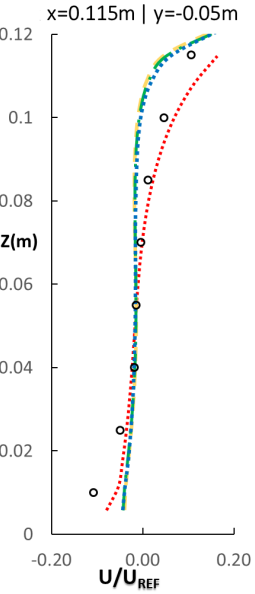

(a)

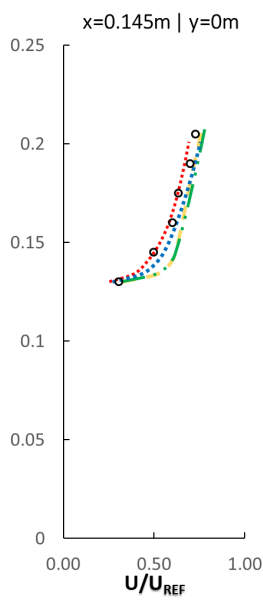

(b)

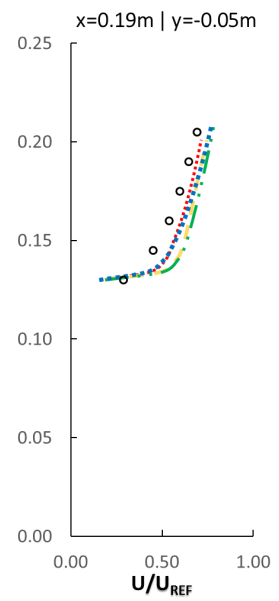

(c)

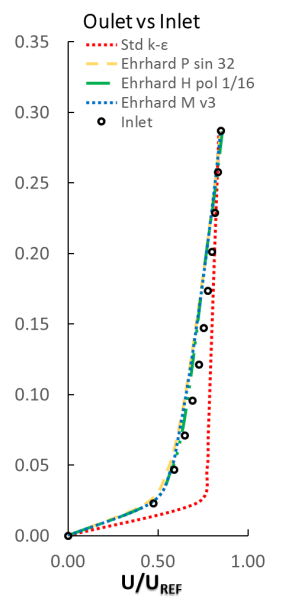

(d)

Figure 21: Comparison of experimental and numerical predictions of non-dimensional velocity at different axial locations of the domain, using std $\mathrm{k}-\epsilon$, Ehrhard applied inside the BIA with both marker and hybrid polynomial blending.

708

show $u$ and $k$ profiles at 5 different locations, $x=0.115 m$ with $y=-0.05 m$, $x=0.145 m$ with $y=0 m, x=0.19 m$ with $y=-0.05 m$ and inlet vs outlet at $y=0 \mathrm{~m}$. From this results it is clear that the proposed models present evident advantages over the standard one. In particular, considering turbulent kinetic energy profiles (Figure 22), both the improved models are capable of fitting with 


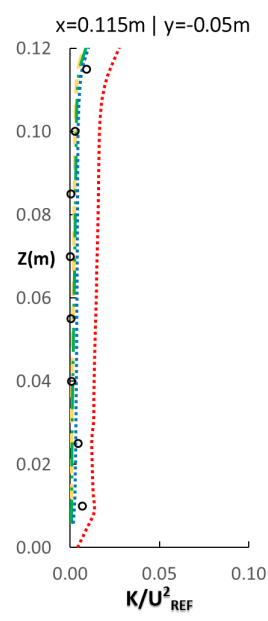

(a)

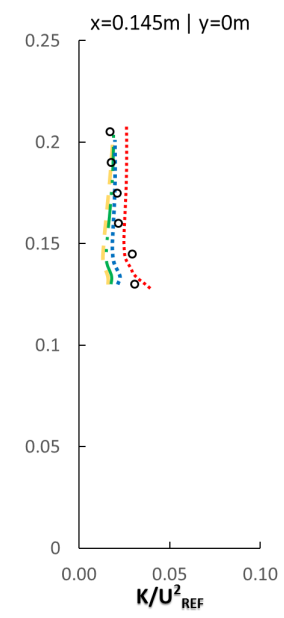

(b)



(c)

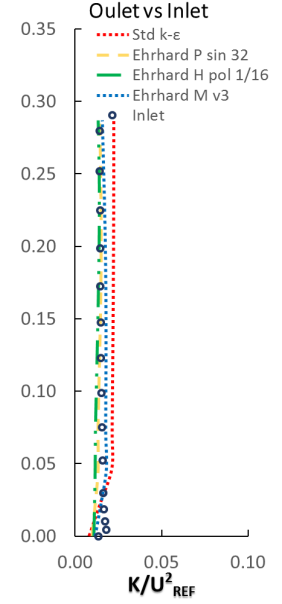

(d)

Figure 22: Comparison of experimental and numerical predictions of non-dimensional turbulent kinetic energy at different axial locations of the domain, using std $k-\epsilon$, Ehrhard applied inside the BIA with both marker and hybrid polynomial blending.

remarkable accuracy the $k$ experimental data, while the standard $k-\epsilon$ approach over-predicts $k$ at almost all the heights. Both the hybrid blending and the marker function coupled with Ehrhard NLEV model, show a reliable behaviour and, even if there are no significant differences between the outcomes of these two approaches, the marker performs slightly better. The velocity field (Figure 21) is narrowly reproduced as well, even if there are still some drawbacks. The most controversial height is $x=0.115 \mathrm{~m}$ with $y=-0.05 \mathrm{~m}$, where the recirculation zone in the very vicinity of the ground, between the blue building and the subsequent obstacle, is misrepresented by the Ehrhard models. The velocity profile at all the other locations are more accurately predicted when applying the proposed models in blending approach; moreover, the inlet velocity profile can be preserved till the outlet only once the improved approach is adopted. Finally, as equally done for the Cedval A1-1 test case, NLEV models have been tested setting the stress-strain coefficients equal to zero: $C_{1}-C_{7}=0$. Once again, due to the limited swirl of the test case, no major differences were ob- 
served in the predictions, suggesting to neglect the higher order terms of the strain-stress relation also for the following simulations, with the aim of further reducing computational costs.

In this regard, a final consideration, valid for both the Cedval A1-1 and B1-1 test cases, concerns the computational cost associated with all the different approaches tested in this study. The computational difference between the standard $k-\epsilon$ model and the comprehensive approach (Table 1) is minimal, related to the addition of both a source term for the $\epsilon$ transport eq. and the variable $C_{\mu}$ formula. As for the blending concept, both the pure and hybrid formulations (being based on a simple turbulence relative deviation) play a minor role in burdening computational cost. The marker function is slightly more onerous than the relative deviation formulas, due to its intrinsic definition which requires both unit velocity vectors and gradients of the streamline-aligned velocity to be locally computed. As for the model applied inside the BIA, NLEV models show a good compromise between accuracy of results and computational costs within the $k-\epsilon$ modelling context ([9] [21] [11]). Moreover, the possibility of neglecting the higher order terms of the stress-strain relation further improves their affordability and feasibility. The $\alpha$ coefficient, whose effect has been explained in Figures 3 and 5 does directly influence the extension of the BIA (the higher the $\alpha$ the more extended the BIA for the sinusoidal transition, vice-versa for the polynomial one), leading, consequently, to different size areas where NLEV models (slightly more expensive than the comprehensive approach) are applied. Translated into a numerical information, setting the scalar residuals limit to $10^{-5}$ (for continuity), this results in a $30 \%$ and $35 \%$ surplus of computational costs for Ehrhard in hybrid blending with polynomial transition $(\alpha=1 / 16)$ and Ehrhard with the marker v.3 concept, in respect to the standard $k-\epsilon$ model. 


\section{Conclusions}

This research stems from the awareness that it is not longer possible to simulate atmospheric boundary layer neglecting the traditional problems affecting the standard RANS two eqs. models: incorrect and approximative representation of velocity and recirculation zones; the extended over-prediction of turbulent kinetic energy; the occurring of horizontal inhomogeneity. The outcomes obtained in this study prove how one possible and valid path for enhancing the overall performance of the $k-\epsilon$ model is the adoption of an improved comprehensive approach valid in open-field, coupled with a Building Influence Area concept. Such an approach can be optimised by properly choosing the physical turbulent quantities (velocity, turbulent kinetic energy) as well as the marker functions controlling the transition; the mathematical form adopted for the latter (sinusoidal, polynomial); the extension of the transition region $(\alpha)$; and the turbulence model form in the Building Influence Area (std $k-\epsilon$, NLEV models). Results for a single and multiple array configuration showed that the best performances are produced using the Ehrhard NLEV model coupled with a hybrid blending and a polynomial transition, or in combination with a marker function indicating the deviation of the actual flow from a parallel one. In particular, the use of the marker function appears interesting as it efficaciously detects the shape and the extension of the BIA without the need for tuning the transition parameter $\alpha$. Moreover, being based on a formulation different from the relative deviation ones, it could be combined with the hybrid blending concept, with the aim of performing a sharper and more effective detection of the BIA. As for the computational costs, all the approaches proposed proved to be feasible and not particularly onerous in respect to the standard $k-\epsilon$. Finally, it must be specified that the main aim of this research is not only centred on the recommendation of a specific turbulence methodology (i.e. NLEV model) over 
a standard one (i.e. std $k-\epsilon$ model) but, in particular, on the development of a methodology/concept permitting a dynamic and multifaceted switch from an undisturbed flow-field simulation to a disturbed one (namely the BIA) within the $A B L$ simulation context. Consequently, the potential evolution of a comprehensive approach and a Building Influence Area appears noteworthy also in relation to other turbulence models, e.g. RNG $k-\epsilon, k-\omega, \mathrm{RSM}$. Future works will further investigate the applicability of this approach to more challenging cases and configuration, such as complex terrains and hazardous dispersion studies, also focusing on full scale cases.

\section{References}

[1] Balogh, M., Parente, A., Benocci, C., RANS simulation of ABL flow over complex terrains applying an Enhanced $k-\epsilon$ model and wall function formulation: Implementation and comparison for fluent and OpenFOAM. Journal of Wind Engineering and Industrial Aerodynamics, 104-106, 2012.

[2] Balogh, M. Numerical simulation of atmospheric flows using general purpose CFD solvers. PhD thesis, Budapest University of Technology and Economics, 2014.

[3] Balogh, M., Parente, A. Realistic boundary conditions for the simulation of atmospheric boundary layer flows using an improved $k-\epsilon$ model. Journal of Wind Engineering and Industrial Aerodynamics144, 183-190, 2015.

[4] Beranek, W., General rules for the determination of wind environment. Proceedings of the 5th international conference on wind engineering, pages 225-234, Fort Collins, Colorado, USA, 1979. 
[5] Bert Blocken, Ted Stathopoulos, and Jan Carmeliet, CFD evaluation of wind speed conditions in passages between parallel buildings-effect of wallfunction roughness modifications for the atmospheric boundary layer flow. Journal of Wind Engineering and Industrial Aerodynamics 95, 941-962, $2007 a$.

[6] Bert Blocken, Ted Stathopoulos, and Jan Carmeliet, CFD simulation of the atmospheric boundary layer: wall function problems. Atmospheric Environment, 41: 238-252, doi: 10.1016/j.atmosenv.2006.08.019. 2007.

[7] Bert Blocken, Introduction to the simulation of atmospheric flows. VKI lecture series, 2017.

[8] Castro, F.A., Palma, J.M.L.M., Silva Lopes, A., Simulation of the askervein flow. part 1: Reynolds averaged navier-stokes equations ( $k-\epsilon$ turbulence model). Boundary-Layer Meteorology 107/3, 501-530, 2003a.

[9] Craft, T.J., Launder, B.E., Suga, K., Development and application of a cubic eddy-viscosity model of turbulence. International Journal of Heat and Fluid Flow, 17: 108-115 Department of Mechanical, Aerospace \& Manufacturing Engineering UMIST, Manchester, UK, 1996.

[10] Durbin, P., Technical note: On the $k-\epsilon$ " Stagnation Point Anomaly. Int. J. Heat Fluid FI. 17: 89-90, 1996.

[11] Ehrhard, J., Moussiopoulos, N., On a new nonlinear turbulence model for simulating flows around building shaped structures. Journal of Wind Engineering and Industrial Aerodynamics, 88: 91-99, 2000.

[12] Fox, D.G., Uncertainty in air quality modeling, Bull. of the Amer. Meteor. Soc., 65, 27-36, 1984. 
[13] Franke J., Hellsten A., Schlunzen H., Carissimo B., Best practice guideline for the CFD simulation of flows in the urban environment-COST Action 732, COST office, 52 pp, 2007.

[14] Franke, J., Hirsch, C., Jensen, A.G., Krus, H.W., Schatzmann, M., Westbury, P.S., Miles, S.D., Wisse, J.A., Wright, N.G., Recommendations on the use of CFD in wind engineering. In: van Beeck, J.P.A.J. (Ed.), COST Action C14, Impact of Wind and Storm on City Life Built Environment. Proceedings of the International Conference on Urban Wind Engineering and Building Aerodynamics, 5-7 May 2004. von Karman Institute, Sint-Genesius-Rode, Belgium.

[15] C. Gorlé, J. van Beeck, P. Rambaud, and G. Van Tendeloo, Cfd modelling of small particle dispersion: The influence of the turbulence kinetic energy in the atmospheric boundary layer. Atmospheric Environment, 43, 673-681, 2009.

[16] C. Gorlé, M. Emory, J. Larsson AND G. Iaccarino, Epistemic uncertainty quantification for RANS modeling of the flow over a wavy wall. Center for Turbulence Research, Annual Research Briefs, 2012.

[17] C. Gorlé, Dispersion of fine and ultrafine particles in urban environment - Contribution towards an improved modeling methodology for Computational Fluid Dynamics. Thesis presented by Catherine Gorlé, von Karman Institute for Fluid Dynamics, 2010.

[18] C. Gorlé, J. Larsson, M. Emory, G. laccarino, The deviation from parallel shear flow as an indicator of linear eddy-viscosity model inaccuracy. Physics of Fluids, 26, 5, 2014.

[19] Hanna, S. R., Confidence limits for air quality model evaluations, as es- 
timated by bootstap and jackknife resampling methods. Atmospheric Environment 23,1385-1398, 1989.

[20] Juretic' F, Kozmar H., Computational modeling of the neutrally stratified atmospheric boundary layer flow using the standard $k-\epsilon$ turbulence model. Journal of Wind Engineering and Industrial Aerodynamics 115:112-120, April 2013.

[21] Lien, F.S., Chen W.L., Leschziner M.A., Low Reynolds-number eddyviscosity modelling based on non-linear stress-strain/vorticity relations. Crete, Greece, 1996.

[22] Logan R.W., Nitta C.K. Comparing 10 methods for solution verification, and linking to model validation. J Aero Comput Inform Commun 3:354-373, 2006.

[23] B. Merci, C. De Langhe, K. Lodefier, E. Dick Axisymmetric Impingement Heat Transfer with a Nonlinear $k-\epsilon$ Model. Journal of Thermophysics and Heat Transfer, Vol. 18, No. 1, 2004.

[24] Parente, A., Longo, R., Ferrarotti, M. CFD boundary conditions, turbulence models and dispersion study for flows around obstacles. VKI lecture series, 2017.

[25] Parente, A., Gorlé , C., van Beeck, J., Benocci, C. Improved k- $\epsilon$ model and wall function formulation for the RANS simulation of $A B L$ flows. Journal of Wind Engineering and Industrial Aerodynamics 99, 267-278, 2011 a.

[26] Parente, A., Gorlé , C., van Beeck, J., Benocci, C., 2011a., A Comprehensive Modelling Approach for the Neutral Atmospheric Boundary Layer: Consistent Inflow Conditions, Wall Function and Turbulence Model. BoundaryLayer Meteorology, 140:411, 2011. 
[27] A. Parente and C. Benocci. On the rans simulation of neutral abl flows. In proceedings of the Fifth International Symposium on Computational Wind Engineering (CWE2010), pages 1-8, Chapel Hill, North Carolina, USA, 2010.

[28] Peralta, C., Parente, A., Balogh, M., Benocci, C., RANS simulation of the atmospheric boundary layer over complex terrain with a consistent $k$ epsilon model formulation. 6th International Symposium on Computational Wind Engineering (CWE2014) Hamburg, Germany, pp. 236-237, 2014.

[29] Pontiggia, M., Derudi, M., Busini, V., Rota, R., Hazardous gas dispersion: A CFD model accounting for atmospheric stability classes. Journal of Hazardous Materials 171 (1-3), 739-747, 2009.

[30] Fernando Porté-Agel, Hao Lu, Yu-Ting Wu, Interaction between large wind farms and the atmospheric boundary layer. Mechanics for the World: Proceedings of the 23rd International Congress of Theoretical and Applied Mechanics, ICTAM2012, 2014.

[31] P. J. Richards and R. P. Hoxey, Appropriate boundary conditions for computational wind engineering models using the k-epsilon turbulence model. Journal of Wind Engineering and Industrial Aerodynamics, 46-47:145 - 153, 1993.

[32] P.J. Richards, S.E. Norris, Appropriate boundary conditions for computational wind engineering models revisited. Journal of Wind Engineering and Industrial Aerodynamics, 99, 257-266, 2011.

[33] Riddle, A., Carruthers, D., Sharpe, 489 A., McHugh, C., Stocker, J., Comparisons between FLUENT and ADMS for atmospheric dispersion modelling and flow around bluff bodies. Atmospheric Environment 38(7), 10291038, 2004. 
[34] Roache P.J. Verification and validation in computational science and engineering. Hermosa Publishers, Albuquerque, NM, 464 pp, 1998.

[35] W. Rodi, Comparison of LES and RANS calculations of the flow around bluff bodies. Journal of Wind Engineering and Industrial Aerodynamics, 6971(0): 55-75. Proceedings of the 3rd International Colloqium on Bluff Body Aerodynamics and Applications, 1997.

[36] Shih, T. H., Zhu, J. and Lumley, J. L., A realizable Reynolds stress algebraic equation model. NASA tech. memo. 105993, 1993.

[37] O'Sullivan, J.P., Archer, R.A., Flay, R.G.J., Consistent boundary conditions for flows within the atmospheric boundary layer. Journal of Wind Engineering and Industrial Aerodynamics 99(1), 65-77, 2011.

[38] Yoshihide Tominaga, Akashi Mochida, Ryuichiro Yoshie, Hiroto Kataoka, Tsuyoshi Nozu, Masaru Yoshikawa, Taichi Shirasawa, AIJ guidelines for practical applications of CFD to pedestrian wind environment around buildings. Journal of Wind Engineering and Industrial Aerodynamics 96, 1749-1761, 2008.

[39] Xie Z, Castro IP Metropolitan Sustainability: Understanding and Improving the Urban Environment. Woodhead Publishing Series in Energy, 2012.

[40] B.W. Yan, Q.S. Li, Y.C. He, P.W. Chan RANS simulation of neutral atmospheric boundary layer flows over complex terrain by proper imposition of boundary conditions and modification on the $k-\epsilon$ model. Environmental Fluid Mechanichs, 16:1-232, 2016.

[41] Yi Yang, Ming Gu, Suqin Chen, and Xinyang Jin. New inflow boundary conditions for modelling the neutral equilibrium atmospheric boundary layer 
930 in computational wind engineering. Journal of Wind Engineering and Indus-

931 trial Aerodynamics, 97(2):88-95, 2009.

${ }_{932}$ [42] Zeman, F. Metropolitan Sustainability: Understanding and Improving the

933 Urban Environment. Woodhead Publishing Series in Energy, 2012.

${ }_{934}$ [43] http://www.mi.zmaw.de/index.php?id=628, CEDVAL at Hamburg Univer${ }_{935}$ sity Compilation of Experimental Data for Validation of Microscale Dispersion Models; WebSite provided by the Environmental Wind Tunnel Laboratory (EWTL) of the Meteorological Institute. 